\title{
ARTICLE
}

\section{TRIM33 switches off Ifnb1 gene transcription during the late phase of macrophage activation}

\author{
Federica Ferri $1,2,3,4,5,6, \star$, Aude Parcelier $1,2,3,4,5, \star$, Vanessa Petit $1,2,3,4,5$, Anne-Sophie Gallouet ${ }^{1,2,3,4,5}$, \\ Daniel Lewandowski ${ }^{1,2,3,4,5}$, Marion Dalloz ${ }^{1,2,3,4,5}$, Anita van den Heuvel ${ }^{7}$, Petros Kolovos ${ }^{7}$, Eric Soler ${ }^{1,2,3,4,7}$, \\ Mario Leonardo Squadrito ${ }^{8}$, Michele De Palma ${ }^{8}$, Irwin Davidson ${ }^{6}$, Germain Rousselet $1,2,3,4,5$ \\ \& Paul-Henri Romeo $1,2,3,4,5$
}

Despite its importance during viral or bacterial infections, transcriptional regulation of the interferon- $\beta$ gene (Ifnb1) in activated macrophages is only partially understood. Here we report that TRIM33 deficiency results in high, sustained expression of Ifnb1 at late stages of toll-like receptor-mediated activation in macrophages but not in fibroblasts. In macrophages, TRIM33 is recruited by PU.1 to a conserved region, the Ifnb1 Control Element (ICE), located $15 \mathrm{~kb}$ upstream of the Ifnb1 transcription start site. ICE constitutively interacts with Ifnbl through a TRIM33-independent chromatin loop. At late phases of lipopolysaccharide activation of macrophages, TRIM33 is bound to ICE, regulates Ifnb1 enhanceosome loading, controls Ifnb1 chromatin structure and represses Ifnb1 gene transcription by preventing recruitment of $\mathrm{CBP} / \mathrm{p} 300$. These results characterize a previously unknown mechanism of macrophage-specific regulation of Ifnb1 transcription whereby TRIM33 is critical for Ifnb1 gene transcription shutdown.

\footnotetext{
${ }^{1}$ CEA/DSV/iRCM/LRTS, 18 route du Panorama, Fontenay-aux-Roses 92265, France. ${ }^{2}$ Inserm U967, Fontenay-aux-Roses 92265, France. ${ }^{3}$ Université Paris-Diderot, Paris 75013, France. ${ }^{4}$ Université Paris-Sud, Orsay 91400, France. ${ }^{5}$ Equipe labellisée Ligue contre le Cancer, 18 route du Panorama, Fontenayaux-Roses 92265, France. ${ }^{6}$ Department of Functional Genomics and Cancer, Institut de Génétique et de Biologie Moléculaire et Cellulaire, CNRS/INSERM/ ULP, BP 163, Strasbourg, Illkirch Cedex 67404, France. ${ }^{7}$ Department of Cell Biology, Erasmus Medical Center, DR Molenwaterplein 50, Rotterdam 3015GE, The Netherlands. ${ }^{8}$ The Swiss Institute for Experimental Cancer Research (ISREC), School of Life Sciences École Polytechnique Fédérale de Lausanne (EPFL), Lausanne CH-1015, Switzerland. * These authors contributed equally to this work. Correspondence and requests for materials should be addressed to I.D. (email: irwin@igbmc.fr) or to G.R. (email: germain.rousselet@cea.fr) or to P.-H.R. (email: paul-henri.romeo@cea.fr).
} 
$\mathrm{n}$ response to viral or bacterial infections, germ line-encoded pattern recognition receptors induce type I interferon (IFN-I, encoded by 14 Ifna and 1 Ifnb genes in mice) gene transcription in myeloid and non-myeloid cells ${ }^{1}$. The secreted type I IFNs act early and transiently during the innate immune response to prime adaptive immunity and then type I Ifn gene transcription is shut down ${ }^{2}$. This transient expression of IFN-I is physiologically important. Whereas early IFN-I expression is important to control infection, pathogens that can overcome this initial control benefit from IFN-I mediated immune system suppression $^{3,4}$.

Among type I Ifn genes, Ifnbl gene regulation has been a model system to study inducible transcription. After pattern recognition receptor-mediated activation, a group of transcription factors, including IRF3/IRF7, NF- $\mathrm{B}$ and ATF2/c-jun, cooperatively binds to a regulatory sequence located between -102 and $-47 \mathrm{bp}$ upstream of the Ifnb1 transcriptional start site (TSS) to form the Ifnb1 enhanceosome. This enhanceosome recruits $\mathrm{CBP} / \mathrm{p} 300$ for acetylation of histone $\mathrm{H} 3$ and permits recruitment of chromatin-remodeling complexes for initiation of transcription ${ }^{5}$.

Monocyte/macrophage-specific regulation of Ifnb1 gene transcription has only been observed in the modulation of the transcriptional activity of IRF3. In monocytes, a PU.1-binding site located in the enhanceosome is constitutively bound by a IRF8/PU.1 myeloid complex that creates a preformed activation complex. This myeloid complex can recruit IRF3 through direct IRF3/IRF8 interaction and is involved in the rapid transcription of Ifnbl observed in monocytes after pathogenic stimulation ${ }^{6}$. Conversely, transcriptional regulator $\mathrm{MafB}$ can impair the interactions between IRF3 bound to the enhanceosome and coactivators, and thus negatively regulates Ifnb1 transcription ${ }^{7}$. Finally, binding of transcriptional regulator YY1 to different sites within the enhanceosome has been shown to result in activation (at the beginning) or repression (at the end) of Ifnb1 gene transcription in fibroblasts ${ }^{8-10}$. Altogether, these results point to the enhanceosome as a regulator of both initiation and termination of the Ifnb1 transcription cycle and of monocyte/ macrophage-specific regulation of Ifnb1 transcription.

The tripartite motif (TRIM) protein family is characterized by a shared N-terminal structure consisting of a RING, two B-box domains, a coiled-coil domain and, for most of the TRIM family members, an E3 ubiquitin ligase activity ${ }^{11}$. TRIM33, together with TRIM24, TRIM28 and TRIM66, form a sub-family of TRIM proteins characterized by a C-terminal plant homeodomain juxtaposed to a bromodomain ${ }^{12}$. These TRIM proteins do not directly bind to DNA but can be recruited by DNA-binding proteins, such as nuclear receptors ${ }^{13}, \mathrm{SMAD}^{14}$, PU.1 or TAL-1 (refs 15,16), to act as transcriptional regulators.

A large number of TRIM proteins are innate immune enhancers and act at multiple levels in signalling pathways, including production and action of IFN- $\beta$ to confer antiviral and antibacterial cytokine production ${ }^{17,18}$. As for the TRIM33 sub-family, TRIM24 represses the viral-defense IFN response in hepatocytes as a consequence of repression of VL30 transcription $^{13,19}$. Although these TRIM24 mediated repressions are potentiated by TRIM33, TRIM33 deficiency alone cannot elicit the IFN response in hepatocytes ${ }^{13,19}$. TRIM24 also acts as a negative regulator of the IFN/signal transducers and activators of the transcription signalling pathway ${ }^{13,19}$, and TRIM33 is essential for cytosolic RNA-induced NLRP3 inflammasome activation ${ }^{20}$. However, up to now, no TRIM protein has been shown to directly regulate $I f n b 1$ gene transcription after recruitment to an If $n b 1$ regulatory sequence.

We have previously shown that TRIM33 can interact with PU.1 to repress its transcriptional activity during haematopoiesis and suggested that TRIM33 may regulate myeloid fate and have a role in macrophages ${ }^{15}$. Here, we show that TRIM33 deficiency in macrophages results in sustained expression of Ifnbl during the late phases of macrophages activation. Our results indicate that TRIM33, recruited by PU.1 on a distal regulatory element of the Ifnb1 gene, regulates Ifnb1 enhanceosome loading and shuts down Ifnbl gene transcription during the late stages of lipopolysaccharide (LPS) activation of macrophages by preventing recruitment of $\mathrm{CBP} / \mathrm{p} 300$.

\section{Results}

TRIM33 controls Ifnb1 expression in activated macrophages. Trim $33^{f l f l}$ mice were crossed with mice expressing the Cre recombinase from the endogenous Lysozyme2 locus (LyzCre) to generate Trim $33^{-/-}$mice. Trim $33^{-/-}$mice did not display developmental abnormalities and were healthy. PCR genotyping of purified haematopoietic cell populations showed deletion of Trim33 in monocytes, bone-marrow-derived macrophages (BMDM), peritoneal macrophages (PMs) and neutrophils but not in myeloid progenitors, nor in B lymphocytes (Fig. 1a, upper panels and Supplementary Fig. 1a). The deletion of the Trim33 gene allele was associated with a loss of TRIM33 expression in BMDM (Fig. 1a, lower panel).

The effects of Trim33 deletion on Ifnb1 expression in macrophages were studied by comparing kinetics of Ifnb1 mRNA levels in wild-type (WT) or Trim $33^{-/-}$BMDM and PM, upon TLR4 activation by LPS or upon TLR3 activation by poly(I:C). These two activation protocols mimic bacterial (LPS) or viral (poly(I:C)) infections. A rapid early increase of Ifnb1 mRNA levels was observed in WT and Trim $33^{-/-}$BMDM or PM activated by LPS (Fig. 1b). Thereafter, Ifnb1 mRNA levels decreased from $2 \mathrm{~h}$ in WT BMDM (or $4 \mathrm{~h}$ in WT PM) and returned to basal levels by $6 \mathrm{~h}$ in WT BMDM and PM. In contrast, although Ifnb1 mRNA levels decreased between 2 and $4 \mathrm{~h}$, Ifnbl mRNA levels began to rise significantly by $6 \mathrm{~h}$ and remained high $24 \mathrm{~h}$ after LPS activation in Trim $33^{-/}-$BMDM or PM (Fig. 1b). This late sustained expression of Ifnbl was also observed when BMDM and PM were stimulated by poly(I:C) with Ifnb1 mRNA levels being much higher at all late time points in poly(I:C) treated Trim33-/- BMDM compared with WT (Supplementary Fig. 1b). The increased Ifnbl mRNA levels in Trim33 ${ }^{-/}-$BMDM activated with LPS correlated with increased levels of secreted IFN- $\beta$ (Fig. 1c). Finally, using a sub-optimal concentration of LPS $\left(0.1 \mathrm{ng} \mathrm{ml}^{-1}\right)$ which did not activate Ifnbl in WT BMDM, we showed, in LPS-activated Trim33-/BMDM, an increased expression of genes whose transcription is dependent on IFN- $\beta$ and occurs late during LPS activation (Fig. 1d). This increased expression was not accounted for by different responses of WT and Trim $33^{-/-}$BMDM to IFN- $\beta$ (Supplementary Fig. 1c) and could be obtained after addition of IFN- $\beta$ during the late phases of LPS activation of WT BMDM (Supplementary Fig. 1d). Altogether, these results show that TRIM33 deficiency is associated with increased Ifnb1 mRNA level associated with IFN- $\beta$ secretion during the late stages of LPS activation of BMDM.

To study if TRIM33 deficiency affects the expression of other Ifn genes, quantitative RT-PCR was performed and showed similar kinetics of Ifna mRNA levels in WT or Trim33-/BMDM with no late increased expression of Ifna mRNA levels in Trim $33^{-/-}$BMDM (Fig. 1e). Finally, highly efficient shRNAmediated depletion of TRIM33 in NIH3T3 cells (NIH3T3 TRIM33- ; Supplementary Fig. 1e) did not modify kinetics of Ifnb1 mRNA levels in response to poly(I:C) (Fig. 1f), indicating cell-specificity in the regulation of Ifnb1 expression by TRIM33.

Potential direct or indirect roles for TRIM33 in Ifnb1 expression were addressed in 'rescue' experiments in which 
a
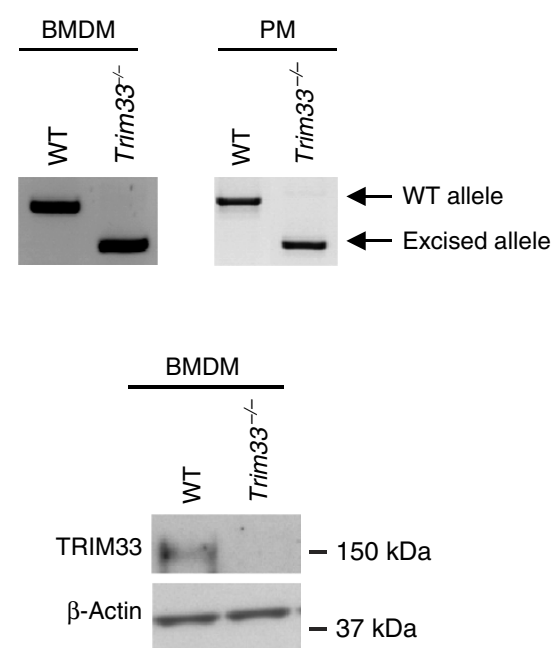

b

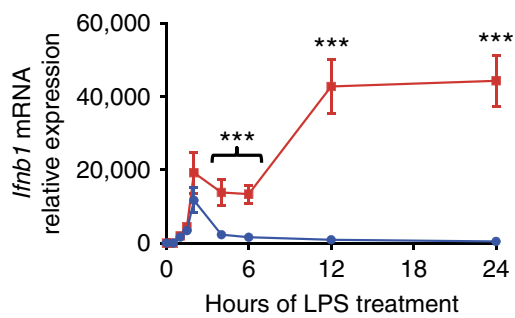

- PM WT

- PM Trim $33^{-/}$

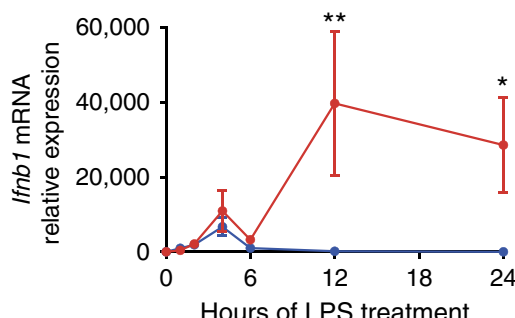

c

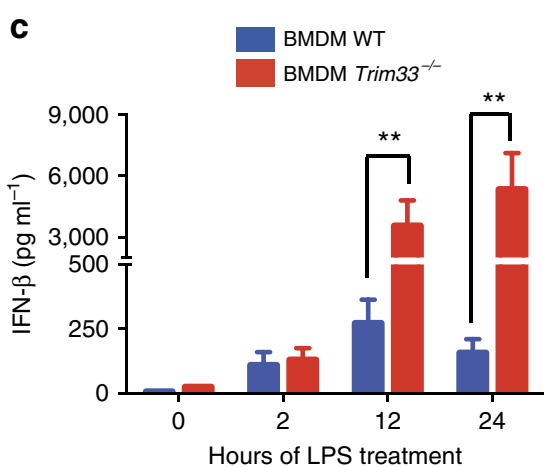

d

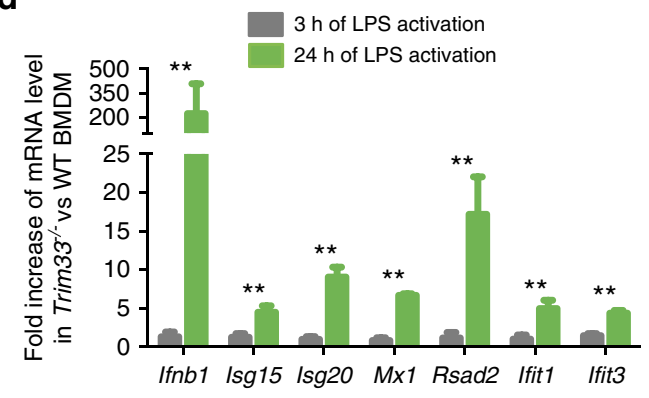

g

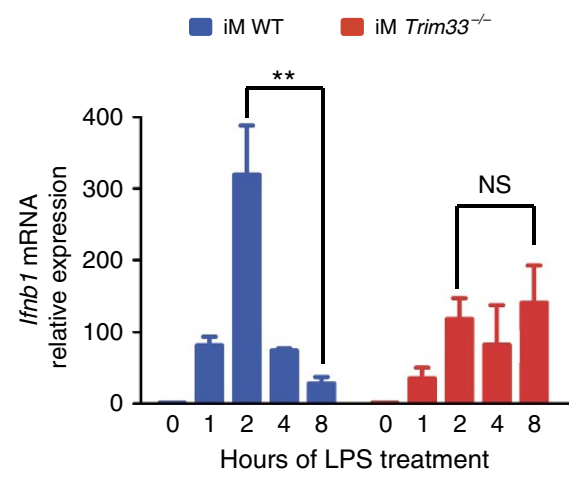

e

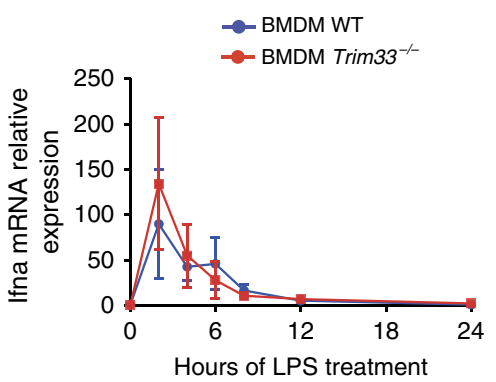

h

f

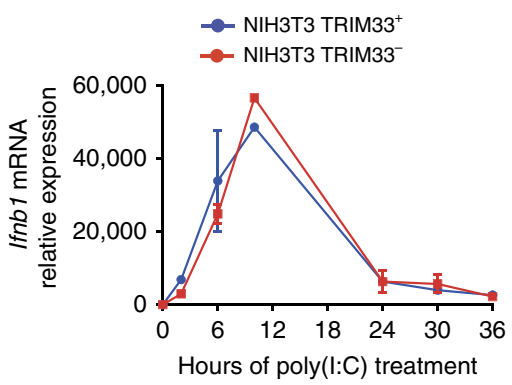

$$
\begin{aligned}
& \text { iM Trim } 33^{--}+\text {GFP } \\
& \text { iM Trim } 33^{-/}+\text {flag-TRIM33 }
\end{aligned}
$$

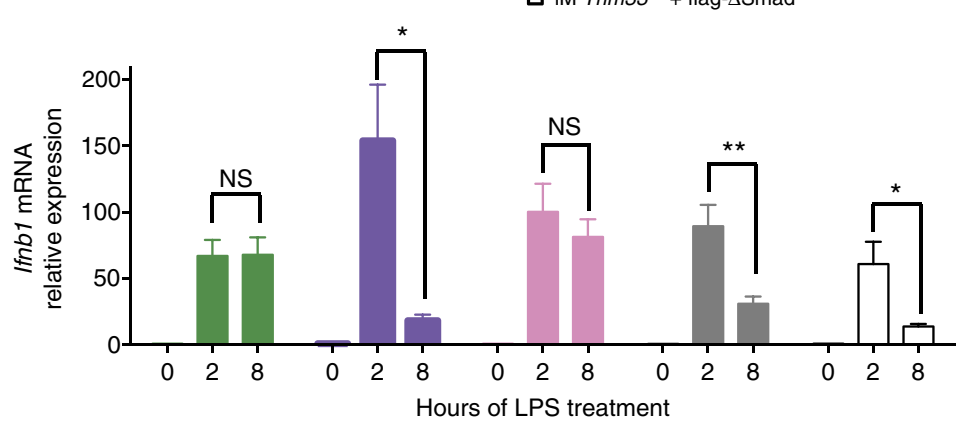

Figure 1 | TRIM33 regulates Ifnb1 gene expression in activated macrophages. (a) Genomic PCR detection of Trim33 in BMDM and PM (top) and western blot analysis of TRIM33 in BMDM from WT and Trim33-/- mice (bottom). (b) Kinetics of Ifnb1 mRNA levels in WT and Trim33-/ - BMDM (left panel) or PM (right panel) after activation with LPS. Mean \pm s.e.m., $n=3$ to 6. (c) IFN- $\beta$ protein levels in supernatants of non-activated and LPS-activated WT and Trim33-/ - BMDM. Mean \pm s.e.m., $n=4$. (d) Relative mRNA levels of Ifnb1 and IFN- $\beta$ target genes in Trim33-/ - BMDM versus WT BMDM treated with LPS $\left(0.1 \mathrm{ng} \mathrm{ml}^{-1}\right)$ for 3 or 24 h. Mean \pm s.e.m., $n=3$. (e) Kinetics of Ifna mRNA levels in WT and Trim33-/ - BMDM activated with LPS. Mean \pm s.e.m., $n=3$. (f) Kinetics of Ifnb1 mRNA levels in NIH3T3 cells expressing a shRNA targeting Trim33 (NIH3T3 TRIM33-) or luciferase (NIH3T3 TRIM33 ${ }^{+}$) and activated with poly(I:C). Mean \pm s.e.m., $n=3$. (g) Kinetics of Ifnb1 mRNA levels in WT and Trim33-/ ${ }^{-}$iM treated for the indicated times with LPS. Mean \pm s.e.m., $n=3$. (h) Relative expression of Ifnb1 mRNA 0, 2 and $8 \mathrm{~h}$ after LPS treatment of Trim $33^{-/}-$iM transduced with a lentivirus coding for GFP (iM Trim33-/ - + GFP), for full-length flag-TRIM33 and GFP (iM Trim33-/- + flag-TRIM33) or for flag-TRIM33 mutants lacking the coiled-coil domain

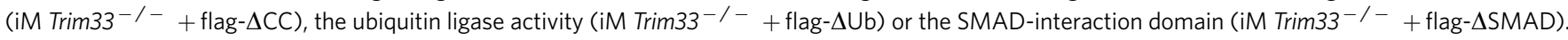
Mean \pm s.e.m., $n=5$. All qRT-PCR data are normalized to HPRT and, unless otherwise indicated, presented relative to expression of untreated WT cells. ${ }^{\star} P<0.05 ;{ }^{\star \star} P<0.01$ and ${ }^{\star \star \star} P<0.001$, Mann-Whitney test. 
TRIM33 protein was added back to LPS-activated immortalized Trim $33^{-1-}$ macrophages $(\mathrm{iM})^{21}$. As anticipated, Trim $33^{-/-}$ $\mathrm{iM}$ bore the expected deletion of Trim33 gene and these cells expressed no TRIM33 protein (Supplementary Fig. 1f). Ifnb1 mRNA levels increased $1 \mathrm{~h}$ after LPS activation of WT and Trim $33^{-/-} \mathrm{iM}$ and peaked at $2 \mathrm{~h}$ with twofold higher observed expression in WT iM than in Trim33-/- iM (Fig. 1g). As anticipated, Ifnbl mRNA levels in WT iM then dropped, whereas in Trim33-1- iM, Ifnb1 mRNA levels remained high (Fig. 1g). Thus, LPS activation of Trim $33^{-/-} \mathrm{iM}$ mimicked the late phase of the Ifnb1 mRNA kinetics seen in Trim33-/- BMDM. To address whether expression of ectopic TRIM33 could rescue Ifnbl downregulation at late time points, a flag-TRIM33-IRES-GFP (or GFP alone) construct was expressed in Trim $33^{-/-} \mathrm{iM}$ and cells were stimulated with LPS (Fig. 1h). The full-length exogenous TRIM33 protein restored Ifnbl downregulation at late time points of LPS activation of Trim $33^{-}$- iM (Fig. 1h) indicating a role for TRIM33 in the regulation of Ifnb1 gene expression during LPS activation of macrophage.

To determine the TRIM33 protein domains involved in Ifnb1 regulation, mutant flag-TRIM33-IRES-GFP constructs lacking the coiled-coil domain (flag- $\Delta \mathrm{CC}$ ), the ubiquitin ligase activity (flag- $\Delta \mathrm{Ub}$ ) or the Smad-interaction domain (flag- $\Delta$ Smad) were expressed in Trim33-1- iM (Supplementary Fig. 1g). The flag- $\Delta \mathrm{Ub}$ and the flag- $\Delta$ Smad rescued normal Ifnb1 expression at late time points of LPS-activated Trim $33^{-/-} \mathrm{iM}$ whereas the flag- $\Delta \mathrm{CC}$ did not rescue normal profile of Ifnb1 expression in activated Trim $33^{-/}-$iM (Fig. 1h). These results indicate that the Smad-interaction and ubiquitin ligase domains are not necessary for TRIM33 regulation of Ifnb1 expression and suggest that TRIM33 is part of a regulatory complex that regulates Ifnb1 transcription.

Altogether, these results reveal a role for TRIM33 in the regulation of Ifnbl expression specifically in downregulating its expression during the late stages of macrophage activation.

TRIM33 binds a distal $\boldsymbol{I} \boldsymbol{f} \boldsymbol{n b} \mathbf{I}$ regulatory element. TRIM33 ChIP-seq data obtained from the RAW 264.7 murine macrophage cell line or from BMDM treated with LPS for $24 \mathrm{~h}$ showed a single TRIM33 peak at the Ifnb1 locus that overlapped with a PU.1 peak located $15 \mathrm{~kb}$ upstream from the Ifnb1 TSS (Fig. 2a). Interestingly, no TRIM33 occupancy was observed at the PU.1 peak located just upstream the Ifnb1 promoter corresponding to the enhanceosome-binding sites. We called this $-15 \mathrm{~kb}$ region the Ifnb1 Control Element (ICE). Recently, the human homologue of ICE, named L2, has been shown to be a virus-inducible enhancer of If $n b 1$ in non-myeloid cells where it displays promoter and enhancer activities ${ }^{22}$.

Constitutive TRIM33 occupancy at ICE was observed in BMDM although occupancy appeared to increase at late time points $(24 \mathrm{~h}$ ) following LPS activation (Fig. $2 \mathrm{~b}$, left panel). PU.1 binding at the Ifnb1 promoter and at ICE was constitutive and detected in WT and Trim33-/- BMDM (Fig. 2b, middle and right panels) indicating that PU.1 binding to ICE was not TRIM33-dependent. Finally, no TRIM33 binding was observed on the Ifnb1 promoter or ICE in NIH3T3 cells (Fig. 2c), indicating macrophage specificity of TRIM33 in binding to ICE.

To determine if TRIM33-mediated regulation of Ifnb1 in myeloid cells can be obtained in NIH3T3 by simultaneous expression of PU.1 and TRIM33, NIH3T3 cells were transduced with a PU.1 expressing lentivirus (Supplementary Fig. 2a). PU.1 expressing NIH3T3 were then transduced with the lentivirus that promoted efficient shRNA-mediated depletion of TRIM33 (Supplementary Fig. 1e) and NIH3T3 PU.1 ${ }^{+}$TRIM33 ${ }^{+}$and
NIH3T3 PU. $1^{+}$TRIM33 ${ }^{-}$responses to poly(I:C) were studied. Whereas TRIM33 deficiency did not modify kinetics of Ifnbl mRNA levels in response to poly(I:C) in normal NIH3T3 cells (Fig. 1f), TRIM33 deficiency increased Ifnb1 mRNA levels in NIH3T3 PU. $1^{+}$cells treated with poly(I:C) but did not result in sustained expression of Ifnb1 during the late stages of poly(I:C) activation (Fig. 2d). These results show that TRIM33 can repress Ifnb1 expression in presence of PU.1 and indicate that additional factors that are not present in NIH3T3 cells are required for TRIM33-mediated late downregulation of Ifnbl expression.

To study the effect of ICE on Ifnbl promoter activity in myeloid cells, we used RAW 264.7 cells in which LPS regulates Ifnb1 gene expression (Supplementary Fig. 2b). RAW 264.7 cells were transfected with luciferase reporter constructs containing a $391 \mathrm{bp}$ fragment of the Ifnb1 promoter preceded by a $408 \mathrm{bp}$ fragment corresponding to the central region of ICE (ICE-Ifnbl Prom-Luc) or the fragment containing the If $n b 1$ promoter alone as a control sequence (Ifnb1 Prom-Luc). Transcriptional activity of ICE-reporter (ICE-Ifnb1 Prom-Luc) showed four to tenfold less activity than the promoter reporter in LPS-activated RAW 264.7 cells indicating that ICE functions as a cis-acting transcriptional repressor element in RAW 264.7 cells (Fig. 2e).

To study the role of ICE in RAW 264.7 and NIH3T3 cells, we used a CRISPR/Cas9 lentiviral system to deliver Cas9 and gRNA (Supplementary Fig. 2c). Using this system, we had to screen 200 RAW 264.7 clones to get one RAW 264.7 clone with monoallelic deletion of ICE (Supplementary Fig. 2d). This low efficiency of recombination in RAW 264.7 cells does not favour the use of transient transfection for CRISPR/Cas9-mediated deletion of ICE, transient transfection efficiency in RAW 264.7 being $15 \%$ compared with $90 \%$ using the lentiviral system. RAW 264.7 and NIH3T3 clones bearing this deletion on one allele (termed RAW ICE ${ }^{+/-}$and NIH3T3 $\mathrm{ICE}^{+/-}$, respectively; Supplementary Fig. 2d) were analysed for Ifnb1 expression after LPS or poly(I:C) activation. In LPS-activated RAW WT cells, Ifnbl mRNA levels increased during the first $4 \mathrm{~h}$ and then gradually decreased (Supplementary Fig. 2e). In contrast, whereas Ifnb1 mRNA levels increased comparably in RAW $\mathrm{ICE}^{+/}$- cells, they subsequently remained stable (Fig. 2f and Supplementary Fig. 2e). In NIH3T3 WT and ICE ${ }^{+} /-$cells activated with poly(I:C), kinetics of Ifnb1 mRNA levels were similar (Fig. $2 \mathrm{~g}$ and Supplementary Fig. 2f). These results indicate that ICE plays a role in the negative regulation of $I f n b l$ expression at late stages of LPS activation in RAW cells.

Sequential deletions centered on the PU.1/TRIM33-binding site of ICE were performed in RAW 264.7 cells using CRISPR/ Cas9 technology (Supplementary Fig. 2g). Of the 96 clones screened, two carried a biallelic deletion of ICE (Supplementary Fig. 2d). Two RAW 264.7 clones bearing a 100 or 176 bp deletion on the two Ifnb1 alleles (termed \#34 and \#15; Supplementary Fig. $2 \mathrm{~g}$ ) were analysed for Ifnb1 expression after LPS or poly(I:C) activation. In the two clones high and sustained IfnbI mRNA levels were found but only after LPS or poly(I:C) activation, with the highest increase being obtained during the late phase of activation (Fig. 2h).

Altogether, these results show that ICE functions as a cis-acting transcriptional repressor element of the Ifn $b 1$ gene activation in macrophages and indicate that TRIM33 and PU.1 bound to ICE may have an important role in Ifnbl gene transcription shutdown during the late phase of macrophage activation.

ICE chromatin structure and interaction with Ifnbl in BMDM. Comparison of reported human and mouse ICE sequences showed 70\% sequence homology around the TRIM33/PU.1 peak with consensus binding motifs for $\mathrm{AP}-1, \mathrm{NF}-\kappa \mathrm{B}$ and IRF 
a

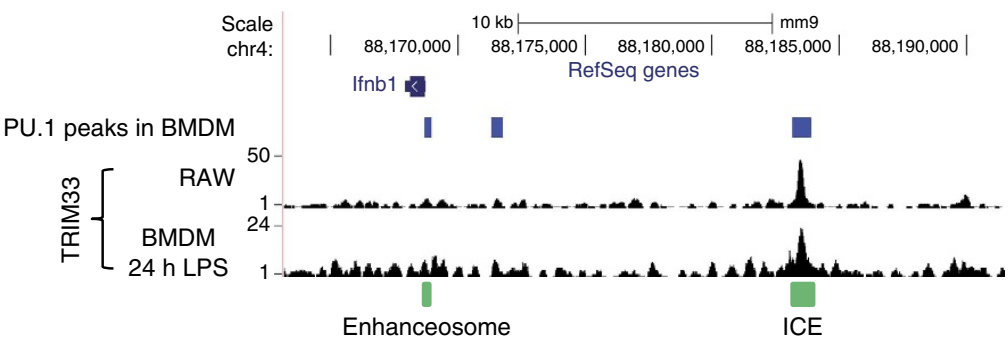

b
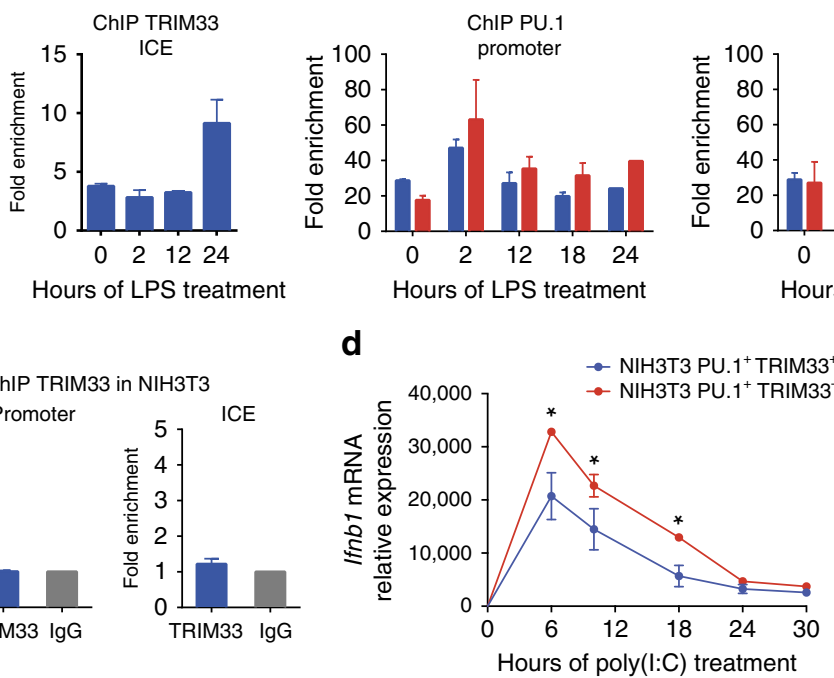

ChIP PU.1
ICE
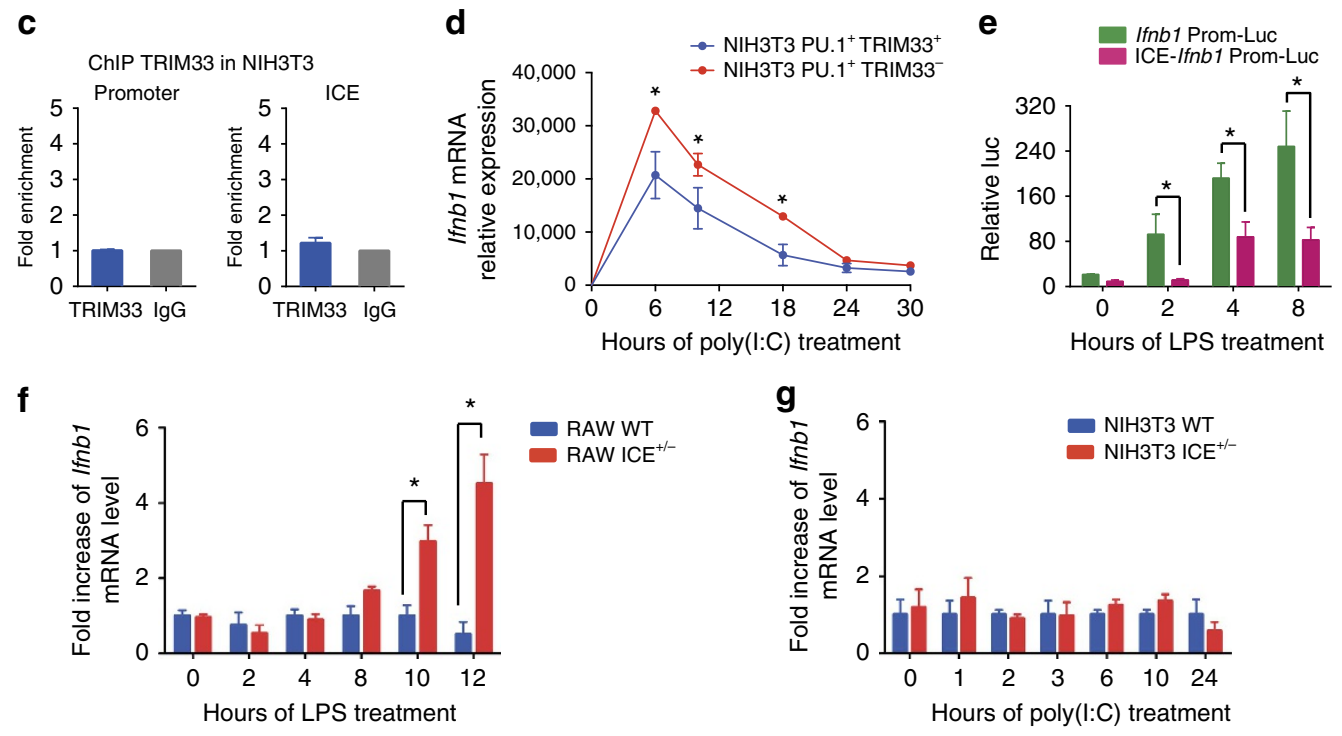

h

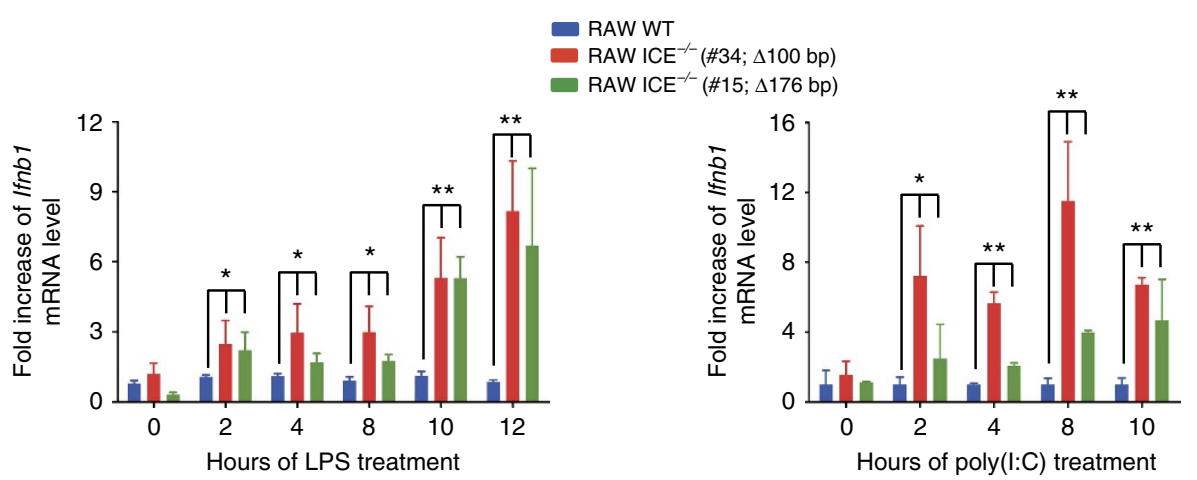

Figure 2 | TRIM33 is bound to a distal Ifnb1 gene regulatory element (ICE) in macrophages. (a) TRIM33 ChIP-seq data in RAW 264.7 cells and BMDM activated for $24 \mathrm{~h}$ with LPS showing TRIM33 binding to ICE, located $15 \mathrm{~kb}$ upstream the Ifnb1 TSS. Blue boxes indicate PU.1 peaks in BMDM (data from ref. 43). Green boxes indicate positions of ICE and the enhanceosome. (b) ChIP-qPCR data for TRIM33 binding on ICE in WT BMDM (left panel) and for PU.1 binding on Ifnb1 promoter and on ICE (right panels) in WT and Trim33-/- BMDM at indicated time points after LPS activation. Data represent the enrichment over a negative control region in the $\beta$-globin promoter. Mean \pm s.e.m., $n=3$. (c) ChIP-qPCR analysis of TRIM33 binding at the Ifnb1 promoter and on ICE in NIH3T3 cells. Mean \pm s.e.m., $n=3$. (d) Kinetics of Ifnb1 mRNA levels in NIH3T3 cells expressing PU.1 and a shRNA targeting Trim33 (NIH3T3 PU. $1^{+}$TRIM33 $^{-}$) or PU.1 and an shRNA targeting luciferase (NIH3T3 PU.1 ${ }^{+}$TRIM33 ${ }^{+}$) and activated with poly(I:C). Mean \pm s.e.m., $n=2$. (e) Luciferase reporter assay in RAW 264.7 cells transfected with reporter constructs containing the Ifnb1 promoter alone (Ifnb1 Prom-luc) or ICE cloned upstream of the Ifnb1 promoter (ICE-Ifnb1 Prom-Luc) and activated with LPS. Mean \pm s.e.m., $n=3$. (f,g) Fold increase of Ifnb1 mRNA levels in ICE $+/-$ versus WT RAW 264.7 cells after LPS activation (f) and in ICE ${ }^{+/-}$versus WT NIH3T3 cells after poly(I:C) activation (g). Mean \pm s.e.m., $n=3$. (h) Fold increase of Ifnb1 mRNA in ICE ${ }^{-/-}$versus WT RAW 264.7 cells after LPS (left) or poly(I:C) (right) activation. Mean \pm s.e.m., $n=3$ to 5 . Clones carrying different deletions at the PU.1/TRIM33 site in ICE are indicated in brackets. ${ }^{\star} P<0.05$ and ${ }^{\star}{ }^{\star} P<0.01$, Mann-Whitney test. 
transcription factors (Supplementary Fig. 3a, left panel). Furthermore, c-JUN, the p65 subunit of NF- $\kappa B$ and IRF3, three transcription factors that form the Ifnbl enhanceosome and regulate its transcriptional activity, were also bound to ICE three hours after LPS activation of BMDM (Supplementary Fig. 3a, right panel; data from ref. 23). We studied a possible TRIM33 dependency of c-jun and p65 binding to ICE but found that TRIM33 deficiency did not modify the kinetics of their binding to ICE (Fig. 3a).

Analysis of ICE chromatin structure in macrophages showed that this region is characterized by high levels of H3K4me3 and $\mathrm{H} 3 \mathrm{~K} 27 \mathrm{ac}$ modification, flanked by regions enriched for H3K4me1, all features of transcriptionally active promoters (Supplementary Fig. 3b, red graph, data from ref. 24). These epigenetic modifications are present in short-term haematopoietic stem cells (ST-HSCs) and in myeloid cells, but not in mature erythroid and lymphoid cells (Supplementary Fig. 3b). We studied the effect of TRIM33 deficiency on the chromatin structure of ICE in nonactivated and LPS-activated BMDM to ask whether TRIM33 has a role in histone modification of this region. Before and during LPS activation of WT and Trim $33^{-/-}$BMDM, no significant differences in H3K4me1 levels on ICE could be detected (Supplementary Fig. 3c). ChIP and ChIP-seq analyses showed a constitutive binding of RNA Polymerase II (Pol II) and constitutively high levels of $\mathrm{H} 3 \mathrm{~K} 4 \mathrm{me} 3$ on ICE (Fig. 3b). Prior to LPS activation, histone $\mathrm{H} 3$ acetylation (H3ac) on ICE was similar in WT and Trim $33^{-/-}$BMDM (Fig. 3c, left panel). During LPS activation, H3ac on ICE did not change in WT BMDM but was transiently increased $10 \mathrm{~h}$ after LPS activation in Trim33-1BMDM (Fig. 3c, left panel). This increased H3ac on ICE in Trim $33^{-/-}$BMDM was associated with transient increase of CBP/ p300 histone H3 acetyl-transferase (HAT) -binding on ICE $10 \mathrm{~h}$ after LPS activation (Fig. 3c, right panel). Collectively, database analyses and our results suggest that, in BMDM, ICE exhibits a promoter-like chromatin signature established early during myeloid differentiation. TRIM33 deficiency did not modify ICE chromatin signature and only led to transient increases of $\mathrm{H} 3 \mathrm{ac}$ and $\mathrm{CBP} / \mathrm{p} 300$ recruitment on ICE whereas a sustained high expression of Ifnb1 was observed in LPS-activated Trim $33^{-/-}$BMDM suggesting that TRIM33 bound to ICE might act at a distance.

To investigate how TRIM33 bound to ICE, that is located $15 \mathrm{~kb}$ upstream of the Ifnb1 transcription unit, may regulate Ifnb1 transcription, chromosome conformation capture experiments followed by deep sequencing (3C-seq) were performed in WT and Trim $33^{-/-}$BMDM before and after LPS activation. ICE (Fig. 3d, top) and the Ifnb1 gene (Fig. 3d, bottom), including its promoter, were used as viewpoints. In WT BMDM, both viewpoints showed a loop between ICE and the Ifnbl gene before LPS activation and a twofold stronger interaction $24 \mathrm{~h}$ after LPS activation (Fig. 3d, blue curves). Interestingly, Trim33 deletion did not significantly modify DNA looping in this region, even after LPS treatment (Fig. 3d, compare red (Trim33-/- ) and blue (WT) curves). These results indicate that ICE interacts with the Ifnb1 proximal region in a constitutive and TRIM33independent manner, but that this interaction is strengthened following LPS stimulation.

TRIM33 represses Ifnb1 by preventing CBP/p300 recruitment. To characterize how TRIM33 might regulate Ifnb1 transcription, we studied the effect of TRIM33 deficiency on temporal binding of p65 and c-jun to the enhanceosome as these two factors are major regulators of its activity. As anticipated, in WT BMDM, c-jun and p65 were recruited to the Ifnb1 promoter at early times following LPS activation $(1 \mathrm{~h})$, but their binding returned to basal levels after $24 \mathrm{~h}$ (Fig. $4 \mathrm{a}$ ). In contrast, in the absence of TRIM33, p65 and c-jun binding to the Ifnbl promoter was maintained even $24 \mathrm{~h}$ after activation (Fig. 4a). Thus, TRIM33 deficiency maintains enhanceosome loading by c-jun and p65 during the late phases of LPS activation.

We then determined the effect of TRIM33 deficiency on Ifnbl transcription and chromatin structure during LPS activation of BMDM. On the Ifnb1 TSS, low levels of paused Pol II were observed before LPS stimulation in both WT and Trim33-/cells (Fig. 4b, upper left panel). In WT BMDM, Pol II occupancy at TSS and in Ifnbl $3^{\prime}$ region increased after $1 \mathrm{~h}$ of stimulation and then was lost at $4 \mathrm{~h}$ (Fig. $4 \mathrm{~b}$, upper panels). In Trim $33^{-/-}$ BMDM, Pol II occupancy similarly increased after $1 \mathrm{~h}$ of stimulation but its levels were maintained up to $24 \mathrm{~h}$ (Fig. $4 \mathrm{~b}$, upper panels). H3K4me3 levels were transiently increased in WT BMDM after $4 \mathrm{~h}$ of LPS stimulation, whereas in Trim $33^{-/-}$ BMDM, persistent and high levels of $\mathrm{H} 3 \mathrm{~K} 4 \mathrm{me} 3$ were also observed up to $24 \mathrm{~h}$ (Fig. $4 \mathrm{~b}$, lower panel and right panel). These observations suggest that the paused Pol II seen in the absence of LPS treatment is used for rapid $I f n b 1$ transcription immediately following LPS stimulation in WT and Trim33-/- BMDM. Then, in WT BMDM, Pol II drops off and Ifnb1 transcription ceases, whereas in Trim33-/- BMDM, continued Pol II occupancy and $\mathrm{H} 3 \mathrm{~K} 4 \mathrm{me} 3$ promote sustained Ifnb1 transcription.

Prior to LPS activation, histone $\mathrm{H} 3 \mathrm{ac}$ on the If $n b 1$ promoter was similar in WT and Trim33-/- BMDM (Fig. 4c). This H3ac increased at $1 \mathrm{~h}$ after LPS activation of WT and Trim $33^{-/-}$ BMDM. It then returned to basal levels in WT BMDM, whereas a 5- to 8 -fold increase in $\mathrm{H} 3 \mathrm{ac}$ was observed after 10 and $24 \mathrm{~h}$ in Trim33-1- BMDM (Fig. 4c). H3ac on the Ifnb1 promoter in LPS-activated Trim33-1- iM mimicked that observed in Trim33-/ - BMDM (Fig. 4d). Expression of ectopic full-length flag-TRIM33 in Trim33-1- iM led to decreased H3ac on the Ifnb1 promoter $7 \mathrm{~h}$ after LPS stimulation (Fig. $4 \mathrm{~d}$ ), whereas the flag- $\Delta \mathrm{CC}$ that could not rescue normal Ifnb1 expression in Trim $33^{-/-} \mathrm{iM}$ (Fig. 1h) did not decrease H3ac on the Ifnb1 promoter $7 \mathrm{~h}$ after LPS stimulation (Fig. 4d). These results indicate a link between TRIM33 and the regulation of $\mathrm{H} 3 \mathrm{ac}$ on the Ifnbl promoter.

Increased $\mathrm{H} 3 \mathrm{ac}$ on the Ifnb1 promoter in LPS-activated Trim33-1- BMDM indicated a possible role for TRIM33 in regulating the equilibrium between recruitment and/or activity of HAT and histone deacetylases (HDAC). Treatment of LPS-activated WT BMDM with trichostatine A, an inhibitor of HDACs, did not result in sustained Ifnbl expression, whereas it did alter expression of genes regulated by HDACs (Fig. 4e, left panel and Supplementary Fig. 4a). Conversely, the increased $\mathrm{H} 3 \mathrm{ac}$ was found to be associated with $\mathrm{CBP} / \mathrm{p} 300 \mathrm{HAT}$ binding on the Ifnbl promoter 10 and $24 \mathrm{~h}$ after LPS activation in Trim33 $-1-$ BMDM (Fig. 4e, right panel). As total CBP protein levels were similar in WT and Trim33-/- BMDM (Fig. 4f), these results indicated that TRIM33 deficiency promotes CBP recruitment. To determine the importance of $\mathrm{CBP} / \mathrm{p} 300$ activity in the phenotype of Trim33- - BMDM, we used C646, a small-molecule inhibitor that efficiently competes with CBP/p300 substrates ${ }^{25}$. Adding C646 $2 \mathrm{~h}$ after LPS induction did not modify Ifnb1 expression in WT BMDM, but reversed the high sustained Ifnb1 transcription level in Trim33-/- BMDMs at both 10 and $24 \mathrm{~h}$ (Fig. $4 \mathrm{~g}$ ). This reversion was associated with decreased levels of H3ac (Fig. 4h) and reduced Pol II occupancy within the Ifnb1 promoter region in Trim $33^{-/-}$BMDM (Supplementary Fig. 4b).

Altogether, these results show that enhanced CBP/p300 recruitment and activity at late times of activation is required for sustained Ifnb1 expression in Trim33-/- BMDM and that TRIM33 may regulate Ifnb1 expression at late times by preventing the recruitment of $\mathrm{CBP} / \mathrm{p} 300$. 
a
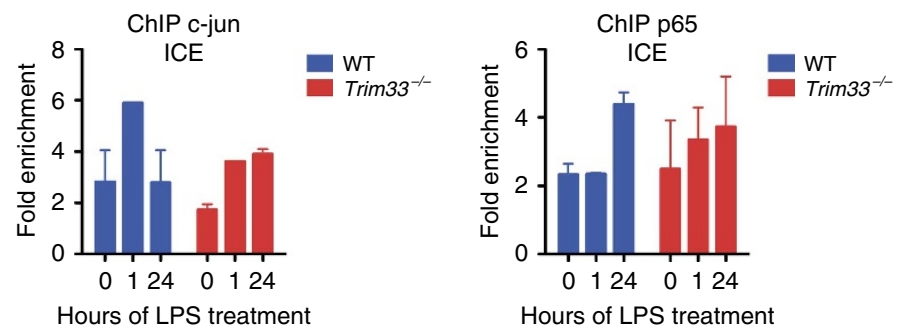

b
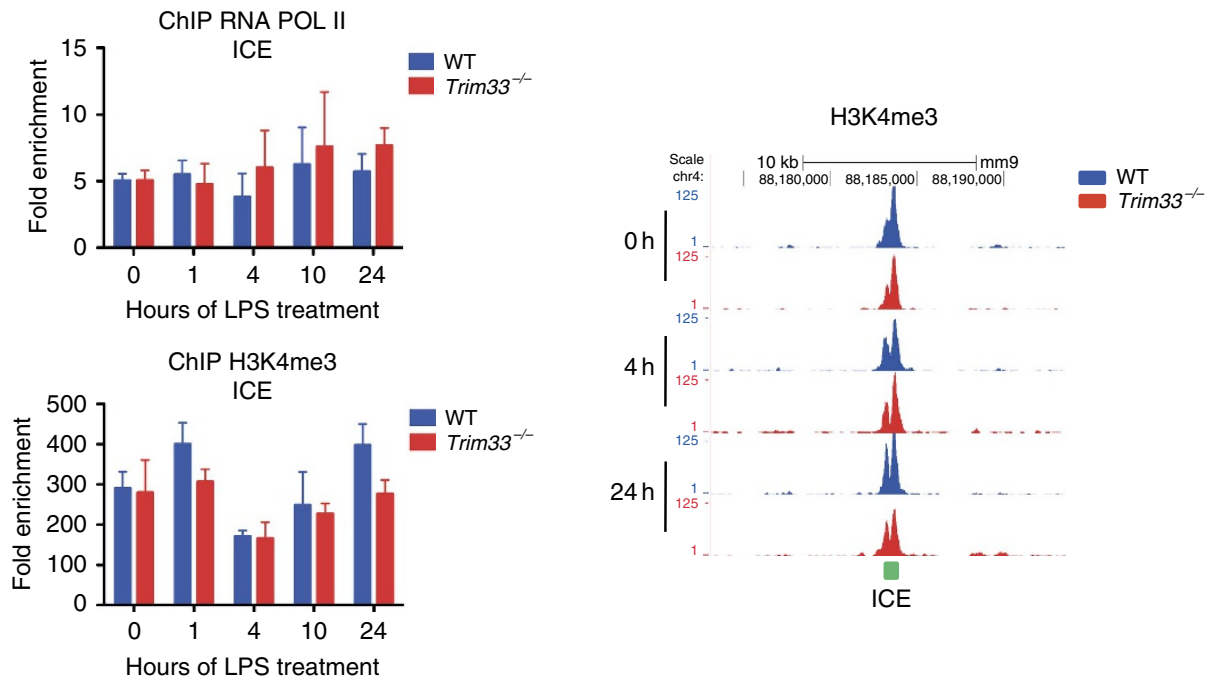

C
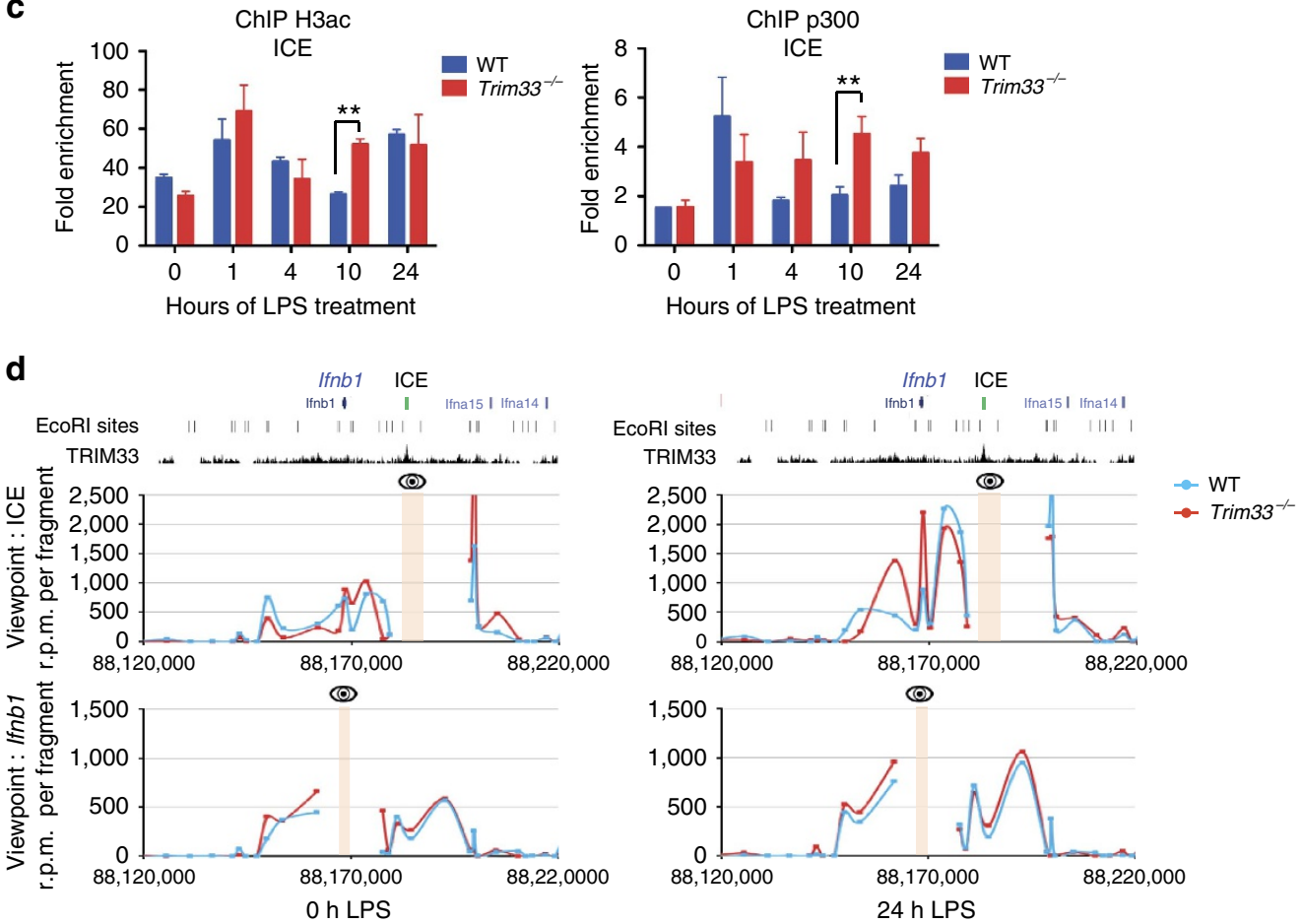

Figure 3 | ICE chromatin structure and interaction with the Ifnb1 promoter during activation of macrophages. (a) ChIP- $q P C R$ analysis of $c-j u n$ or p65 binding at ICE in WT and Trim33-/- BMDM treated for the indicated times with LPS. Mean \pm s.e.m., $n=2$ to 4 . (b) ChIP-qPCR analysis of RNA Pol II (upper left panel) and H3K4me3 (lower left panel) at ICE in WT and Trim33-/ - BMDM treated for the indicated times with LPS. Mean \pm s.e.m., $n=3$ to 5 . (Right panel) UCSC genome browser images showing H3K4me3 ChIP-seq profiles at ICE in WT and Trim33-/- BMDM treated for the indicated times with LPS. (c) ChIP-qPCR analysis of acetylated histone H3 (left panel) and CBP/p300 (right panel) at ICE in WT and Trim33-/ - BMDM treated for the indicated times with LPS. Mean \pm s.e.m., $n=4 .{ }^{\star \star} P<0.01$, Mann-Whitney test. (d) DNA looping at the Ifnb1 locus was determined by $3 C$-seq performed before or $24 \mathrm{~h}$ after LPS activation of WT and Trim33-/- BMDM using either ICE (upper panel) or Ifnb1 gene (lower panel) as viewpoints (shown by an eye and a yellow band). Data represent normalized reads per million (r.p.m.) per restriction fragment. The $x$ axis shows the genomic coordinates of the Ifnb1 locus. The positions of the EcoRI restriction sites and the TRIM33 ChIP-seq profile in BMDM are indicated on the top. 


\section{Discussion}

Although IFN- $\alpha$ and IFN- $\beta$ share the same receptor, IFN- $\beta$ seems to be the major immune-suppressive IFN $^{26}$ and macrophagerestricted synthesis of IFN- $\beta$ can contribute to protective or pathological immune responses in the lung ${ }^{27}$. Surprisingly, despite the importance of IFN- $\beta$ production by macrophages, no cis- or trans-acting factor that might confer a myeloid specificity to Ifnb1 gene transcription has been characterized. The data presented in this study provide evidence of a previously unappreciated regulation of Ifnbl transcription in macrophages

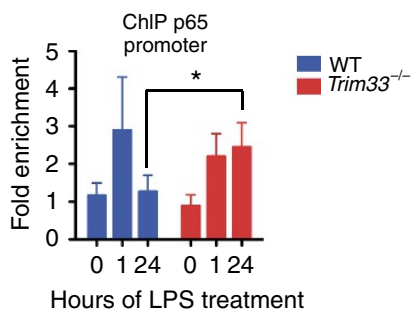

b
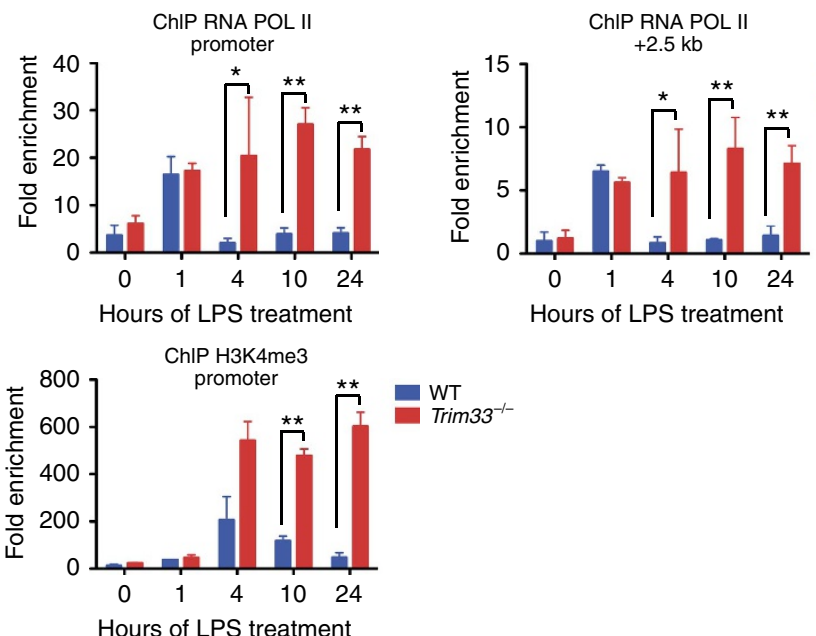

WT

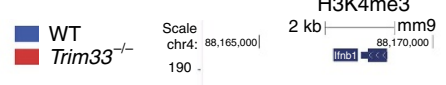

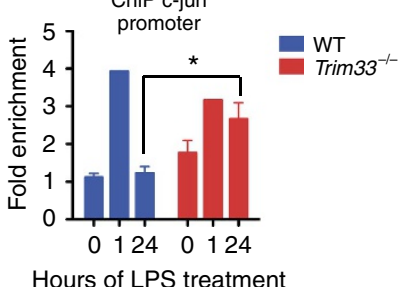

Hours of LPS treatment
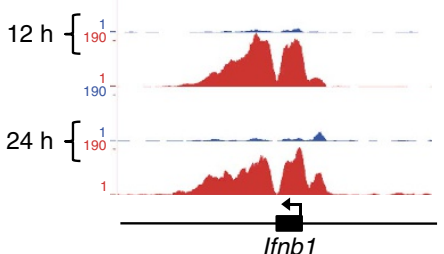

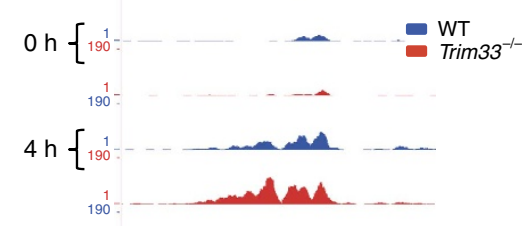

c
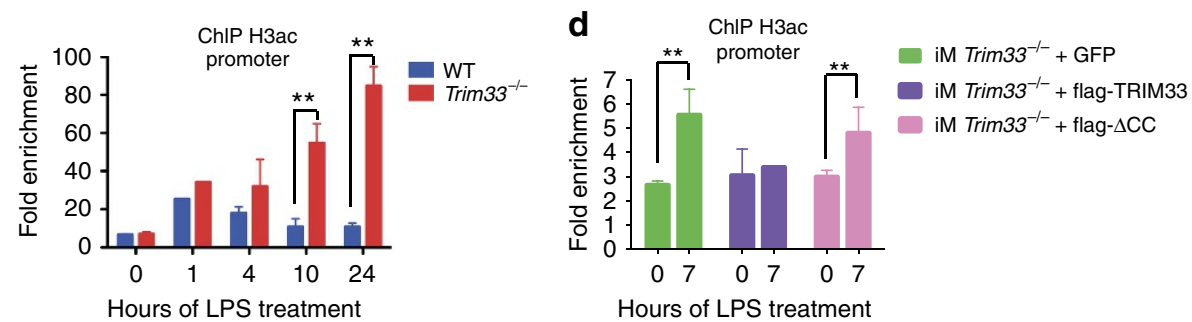

e
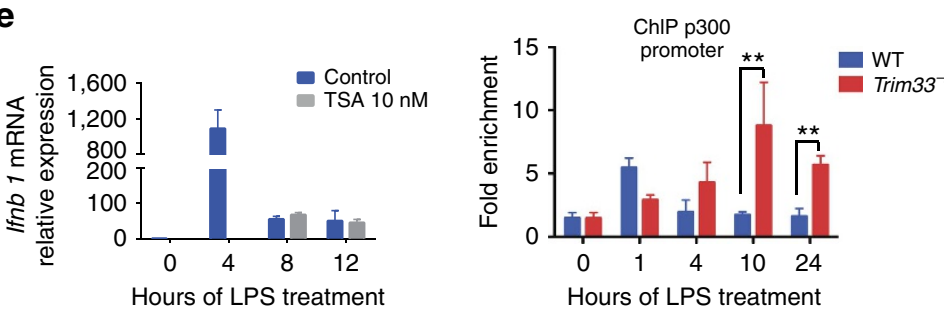

f

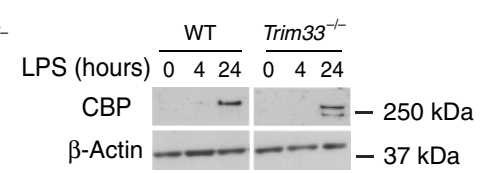

g

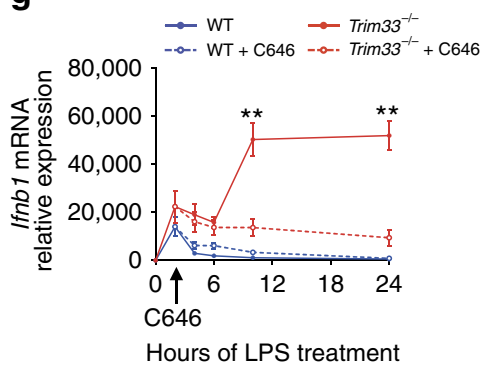

h

h $\quad$ WT $\quad$ Trim $33^{-1-}$

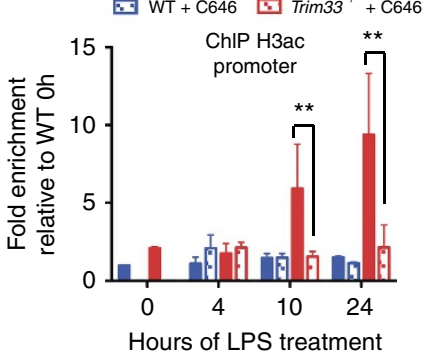

i

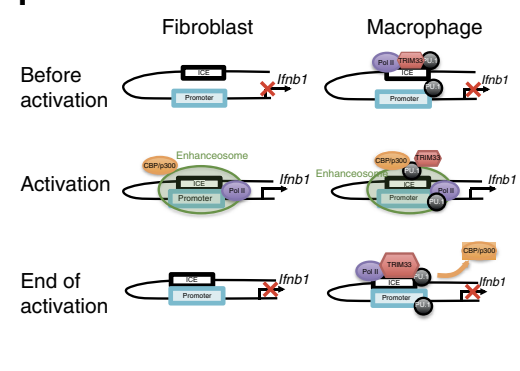


through a macrophage-restricted TRIM33 recruitment on a regulatory sequence, designated as ICE, located $15 \mathrm{~kb}$ upstream from the Ifnbl TSS.

ICE has an open-chromatin structure highly conserved during myeloid differentiation and characterized by Pol II occupancy and tri-methylation of $\mathrm{H} 3 \mathrm{~K} 4$, two features that are not typical marks of an enhancer or a repressor. In addition to a PU.1binding site, ICE contains DNA-binding sites for transcriptional regulators such as IRF3, c-jun or p65 that are also part of the enhanceosome. ICE has been recently shown to interact with the Ifnb1 promoter in human fibroblasts in the absence of any viral infection $^{22}$. Here, we show that ICE can also interact with the Ifnb1 promoter in non-activated macrophages. These combined findings indicate that ICE/Ifnb1 loop is cell-type independent, stable and independent of viral or bacterial stimulation. Such enhancer or repressor loop stability has been described during development where they are associated with paused polymerase ${ }^{28}$ and in human fibroblasts where TNF- $\alpha$-responsive enhancers contact their target promoters before TNF- $\alpha$ activation ${ }^{29}$.

Transient transfection in fibroblast cell lines shows that ICE acts as an enhancer of Ifnbl promoter activity, but only after viral infection $^{22}$. In contrast, in macrophages, ICE acts as a repressor of Ifnb1 promoter activity during LPS activation. Furthermore, the high and sustained expression of Ifnb1 in LPS- or poly(I:C)activated RAW cells where both alleles of ICE are deleted using lentiviral delivery of Cas9 and gRNA indicates that ICE has a repressor activity when these cells are stimulated. Although the lentiviral system used might increase the frequency of off-target effects $^{30,31}$, the similar results obtained with three different clones and with different gRNAs make off-target effects less likely. Use of recombinant Cas9 protein ${ }^{32}$ or highly efficient transient transfection of RAW cells will reduce off-target effects and definitively show that ICE is a unique element that acts as an enhancer in fibroblasts, but displays promoter marks and acts as a repressor at late stages of LPS activation of macrophages. These opposite roles of ICE in fibroblasts and macrophages might be linked to recruitment of distinct proteins in the two cell types. Indeed, PU.1, which is not expressed in fibroblasts, is bound to ICE in macrophages and might be involved in the cell-specific function of ICE. Ectopic PU.1 expression in TRIM33 ${ }^{+}$and TRIM33 - NIH3T3 cells indicates that TRIM33-mediated repression of $I f n b 1$ is dependent on PU.1 expression. However, in fibroblasts activated with poly(I:C), PU.1 expression associated with TRIM33 deficiency does not result in the sustained Ifnb1 expression observed in poly(I:C)-activated Trim33-/- BMDM. This result suggests that factors present in BMDM, but not in fibroblasts, are required for Ifnb1 repression during the late stages of BMDM activation. Amongst haematopoietic-restricted PU.1 partners, IRF8 and IRF3 that regulate Ifnb1 activation in
BMDM $^{6,33}$ are good candidates to cooperate with TRIM33 for Ifnb1 repression during the late stages of BMDM activation.

Several TRIM proteins regulate production and action of IFN- $\beta$ during innate immune response. TRIM6 is a positive regulator of IFN- $\beta$ and is critical for IFN- $\beta$-mediated antiviral response ${ }^{34}$, TRIM25 is essential for RIG-I-mediated-type I IFN induction ${ }^{35}$, TRIM21 regulates IRF3-dependent IFN- $\beta$ expression $^{36}$ and TRIM56 modulates STING and subsequent IFN- $\beta$ induction ${ }^{37}$. Here, we show the implication of TRIM33 in the transcriptional regulation of Ifnb1. Interestingly, TRIM33 deficiency did not alter the initial activation of $I f n b 1$ transcription after LPS activation of macrophages but impaired repression of Ifnbl expression during the late stages of this activation. Together with the continuous binding of TRIM33 on ICE, the complementation of the Ifnbl phenotype in macrophages by reexpression of TRIM33 in Trim33-/ - immortalized macrophages suggested a direct role of TRIM33 in Ifnb1 transcription and indicated that the activity and/or substrate availability of TRIM33 regulated Ifnbl expression at the end of LPS activation of macrophages.

Two lines of evidence suggest that, during the late stages of LPS activation of BMDM, TRIM33 represses Ifnb1 expression through modulation of $\mathrm{CBP} / \mathrm{p} 300$ recruitment and/or activity at the If $n b 1$ promoter. First, TRIM33 inactivation led to recruitment of $\mathrm{CBP} / \mathrm{p} 300$ at the Ifnb1 promoter and enhanced H3ac. Second, $\mathrm{CBP} / \mathrm{p} 300$ activity is critical for enhanced Ifnb1 transcription at late stages as chemical inhibition of $\mathrm{CBP} / \mathrm{p} 300$ enzymatic activity could restore the regulated expression of $I f n b 1$ gene in Trim $33^{-/-}$macrophages. As $\mathrm{CBP} / \mathrm{p} 300$ acts as a switch to initially turn on $I f n b 1$ gene transcription ${ }^{38}$, these results indicated that TRIM33 might turn off Ifnb1 transcription during the late stages of LPS activation of macrophages by impairing the $\mathrm{CBP} / \mathrm{p} 300$ recruitment and activity at the Ifnb1 promoter. This TRIM33-dependent recruitment and activity of $\mathrm{CBP} / \mathrm{p} 300$ could be direct and/or mediated by trans-acting factors such as p65 that are, in Trim $33^{-/-}$BMDM, loaded onto the enhanceosome during the late phases of LPS activation. However, as PU.1 can interact with $\mathrm{CBP} / \mathrm{p} 300$ (refs 39,40) and TRIM33, we propose a model where CBP/p300 and TRIM33 compete for PU.1 binding during LPS activation of BMDM. During the initial stages of LPS activation, $\mathrm{CBP} / \mathrm{p} 300$ recruited by the enhanceosome might activate Ifnb1 transcription, whereas during the late stages of LPS activation, TRIM33 might interact with PU.1, impair PU.1/CBP/ p300 interaction and shut down Ifnb1 transcription (Fig. 4i).

Numerous studies have shown the complexity of Ifnbl gene transcriptional regulation. Our data describe a new regulatory layer through identification of cis- and trans-acting elements that regulate $I f n b 1$ gene transcription at specific steps of macrophage activation, but not in fibroblasts. These findings not only provide

Figure 4 | TRIM33 regulates Ifnb1 gene expression through inhibition of CBP/p300 recruitment. (a) ChIP-qPCR of p65 (left panel) and c-jun (right panel) at the Ifnb1 promoter in WT and Trim33-/- BMDM treated with LPS. Mean \pm s.e.m., $n=2$ to 4 . (b, top) ChIP-qPCR of RNA Pol II at the Ifnb1 promoter (left panel) and at a region located $+2.5 \mathrm{~kb}$ from TSS (middle panel) in WT and Trim33-/ - BMDM treated with LPS. Mean \pm s.e.m., $n=3$ to 5 . (Bottom) ChIP-qPCR (left panel) and ChIP-seq (right panel) of H3K4me3 at the Ifnb1 promoter in WT and Trim33-/- BMDM treated with LPS

Mean \pm s.e.m., $n=3$. (c) ChIP-qPCR of H3ac at the Ifnb1 promoter in WT and Trim33- ${ }^{-}$BMDM treated with LPS. Mean \pm s.e.m., $n=4$. (d) ChIP-qPCR of H3ac at the Ifnb1 promoter in Trim33-/ ${ }^{-1 M}$ expressing GFP (iM Trim33-/ + GFP), full-length flag-TRIM33 and GFP (iM Trim33-/ - + flag-TRIM33), or flag-TRIM33 lacking the coiled-coil domain and GFP (iM Trim33-/ + flag- $\Delta C C$ ), and treated for 0 or $7 \mathrm{~h}$ with LPS. Mean \pm s.e.m., $n=3$. (e, left) Relative expression of Ifnb1 mRNA in LPS-activated WT BMDM in presence or absence of Tricostatin A (TSA), added $4 \mathrm{~h}$ after LPS stimulation. Mean \pm s.e.m., $n=3$. (Right) ChIP-qPCR of CBP/p300 binding at the Ifnb1 promoter in WT and Trim33-/- BMDM treated with LPS. Mean \pm s.e.m., $n=4$. (f) Western blot of CBP in WT and Trim33-/- BMDM treated with LPS. $\beta$-actin is shown as control. $n=4$ independent experiments. (g) Kinetics of Ifnb1 mRNA levels in LPS-activated WT and Trim33-/- BMDM, in the presence (dotted lines) or absence (continuous lines) of C646. C646 was added $2 \mathrm{~h}$ after LPS stimulation and Ifnb1 mRNA levels were determined. Mean \pm s.e.m., $n=8$. (h) ChIP-qPCR of H3ac at the Ifnb1 promoter in LPS-activated WT and Trim33-/ - BMDM, in the presence (hatched columns) or absence (solid columns) of C646. Data are presented relative to H3ac binding in untreated WT BMDM. Mean \pm s.e.m., $n=2$. (i) Schematic representation depicting the role of TRIM33 in the macrophage-restricted Ifnb1 gene transcription shut down. ${ }^{\star} P<0.05$ and ${ }^{* \star} P<0.01$, Mann-Whitney test. 
important insights into the cell-specific regulation of Ifnb1 gene transcription but also extend the role of TRIM proteins as critical regulators of the innate immune response.

\section{Methods}

Mice. To generate deletion of Trim33 in mature myeloid cells, Trim $33^{\mathrm{fl} / \mathrm{fl}}$ C57Bl/6-CD45.2 mice ${ }^{15}$ were crossed with Lysozyme-Cre C57Bl/6-CD45.2 mice

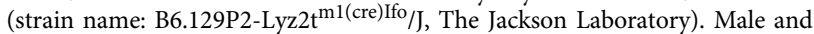
female mice of 8-12 months of age were used. Experiments were performed in compliance with European legislation and with the Ethics Committee of the French Ministry of Agriculture (Agreement B9203202).

Cell culture. For BMDM generation, mouse bone marrow (BM) was flushed out of the tibiae, femora and humeri using a syringe with PBS, filtered through a $70-\mu \mathrm{m}$ mesh filter (Becton Dickinson) to remove debris and pelleted by centrifugation. Adherent cells were removed by incubating BM cells on culture dishes for $3 \mathrm{~h}$ in IMDM supplemented with $10 \%$ foetal calf serum (FCS), $1 \%$ penicillin/streptomycin (PS; Life Technologies) and $10 \mu \mathrm{M}$ thioglycerol (Sigma Aldrich), then non-adherent cells were cultured for 7 days on culture dishes in the same medium supplemented with $25 \mathrm{ng} \mathrm{ml}^{-1}$ mouse CSF1 (Miltenyi Biotec). On day 7, BMDM were activated with LPS or poly(I:C) in IMDM supplemented with $2.5 \%$ FCS and $1 \%$ PS. For PMs, cells were collected with cold PBS and cultured for $2 \mathrm{~h}$ in RPMI 1640 medium (Life Technologies), supplemented with 10\% FCS. All adherent cells expressed F4/80 and were considered as PMs. PM were activated with LPS or poly(I:C) in RPMI 1640 medium supplemented with $2.5 \%$ FCS and $1 \%$ PS. WT and Trim33 $33^{-/-}$iM were generated as previously described ${ }^{21}$. Briefly, BM cells isolated from WT and Trim $33^{-/-}$mice were cultured in IMDM supplemented with $10 \%$ FCS and $50 \mathrm{ng} \mathrm{ml}^{-1}$ mouse CSF1 (Peprotech). After overnight culture, the cells were transduced with a lentiviral vector expressing the SV40 large

$\mathrm{T}$ antigen (SFFV.Tag LV). Cells were then cultured in a chemically defined medium (SFM-macrophage medium, Life Technologies) supplemented with CSF1

$\left(50 \mathrm{ng} \mathrm{ml}^{-1}\right)$. Single clones were isolated that expressed F4/80, CD11b but not Gr1. The resulting clones, termed iM, were maintained in IMDM supplemented with $20 \%$ foetal bovine serum, $1 \%$ PS and $50 \mathrm{ng} \mathrm{ml}^{-1} \mathrm{CSF} 1$. iM were activated with LPS in IMDM supplemented with 2.5\% FCS and 1\% PS. For cell lines, RAW 264.7 and NIH3T3 cells were grown in DMEM (Life Technologies) supplemented with $10 \%$ FCS and 1\% PS. RAW 264.7 cells were activated with LPS in DMEM supplemented with $1 \%$ FCS. Unless otherwise indicated, LPS (Sigma Aldrich) was used at $100 \mathrm{ng} \mathrm{ml}^{-1}$, poly(I:C) at $30 \mu \mathrm{g} \mathrm{ml}^{-1}$, TSA (Sigma Aldrich) at $10 \mathrm{nM}$, and C646 (Merck Millipore) at $10 \mu \mathrm{M}$. NIH3T3 were transfected with $10 \mu \mathrm{g} \mathrm{ml}^{-1}$ poly(I:C) (Sigma Aldrich)

Chromatin Immunoprecipitation (ChIP). Cells were fixed with $1 \%$ formaldehyde for $10 \mathrm{~min}$ at $37^{\circ} \mathrm{C}$, lysed in SDS lysis buffer (50 mM Tris pH8, $10 \mathrm{mM}$ EDTA, $1 \%$ SDS, protease inhibitor cocktail (Roche)) and sonicated. Supernatant was diluted 10 times in IP dilution buffer $(16.7 \mathrm{mM}$ Tris pH8, $167 \mathrm{mM} \mathrm{NaCl}, 1.2 \mathrm{mM}$ EDTA, $1.1 \%$ TritonX-100, 0.01\% SDS, protease inhibitor cocktail (Roche)) and immunoprecipitations were carried out overnight with specific antibodies. Immunoprecipitated chromatin was collected using Protein A Agarose/Salmon Sperm DNA beads (Millipore) and, after washing and elution, reverse cross-linking was carried out with $0.2 \mathrm{M} \mathrm{NaCl}$ at $65^{\circ} \mathrm{C}$ overnight. The chromatin was then digested by $20 \mu \mathrm{g}$ of Proteinase $\mathrm{K}$ (Invitrogen) for $1 \mathrm{~h}$ at $45^{\circ} \mathrm{C}$ and isolated by phenol-chloroform extraction. PCR reactions were performed using SYBR Green PCR Master Mix (Applied Biosystems) and specific primers sequences are listed below.

For sequencing, $10 \mathrm{ng}$ of purified DNA from ChIP was adapter-ligated, PCR amplified and sequenced on the Illumina Genome Analyzer IIx as single-end 50 base reads following Illumina's instructions. Sequence reads were mapped to reference genome mm9/NCBI37 using Bowtie v0.12.7.

3C-seq. 3C-seq was performed as previously described ${ }^{41}$, using EcoRI as first cutter and DpnII as second cutter. Briefly, cells were fixed with $2 \%$ formaldehyde for $10 \mathrm{~min}$ at room temperature, and nuclei were prepared by lysing cells in $10 \mathrm{mM}$ Tris $\mathrm{pH} 8,10 \mathrm{mM} \mathrm{NaCl}, 0.2 \% \mathrm{NP}-40$. Nuclei were lyzed in $0.5 \mathrm{ml} 1 \times$ EcoRI buffer supplemented with $0.3 \%$ SDS for $1 \mathrm{~h}$ at $37^{\circ} \mathrm{C}$. Triton X-100 was added at $2 \%$ final for another $1 \mathrm{~h}$ at $37^{\circ} \mathrm{C}$. DNA was then digested with 400 units of EcoRI overnight at $37^{\circ} \mathrm{C}$. Samples were incubated for $25 \mathrm{~min}$ at $65^{\circ} \mathrm{C}$ with $1.6 \%$ SDS, diluted to $7 \mathrm{ml}$ with ligase buffer, supplemented with $1 \%$ Triton $\mathrm{X}-100$ and incubated at $37^{\circ} \mathrm{C}$ for $1 \mathrm{~h}$. Ligation was performed for $4 \mathrm{~h}$ at $16^{\circ} \mathrm{C}$ with $100 \mathrm{u} \mathrm{T} 4 \mathrm{DNA}$ ligase, then $300 \mu \mathrm{g}$ proteinase $\mathrm{K}$ were added and samples were decrosslinked overnight at $65^{\circ} \mathrm{C}$. RNase $(300 \mu \mathrm{g})$ was added for $45 \mathrm{~min}$ at $37^{\circ} \mathrm{C}$, and samples were extracted with phenol/chloroform/isoamyl alcohol (25:24:1; PCI). After precipitation, samples were digested with DpnII, extracted with PCI and precipitated, and ligated in $15 \mathrm{ml}$ ligation buffer at $16^{\circ} \mathrm{C}$ for $4 \mathrm{~h}$ before another PCI extraction and precipitation. Samples were amplified by PCR with the primers listed below, and PCR products were purified on QIAprep columns (Qiagen). The resulting libraries were sequenced on a Illumina HiSeq 2000 as single-end 100 bp reads. After trimming, reads were aligned on the $\mathrm{mm} 9$ version of the mouse genome using Bowtie.
Results are presented as the number of reads per million reads per EcoRI fragment, in the middle of each fragment.

Assay for IFN- $\boldsymbol{\beta}$. WT and Trim $33^{-/-}$BMDM supernatants were collected after LPS stimulation. ELISA was performed with LegendMax Mouse IFN- $\beta$ kit (Biolegend), according to manufacturer's instructions.

Quantitative RT-PCR. Total RNA was extracted with the RNeasy Plus Micro kit (Qiagen) and reverse transcribed with random primers and Superscript III (Life Technologies). Quantitative PCR was performed using the Power SYBR green PCR master mix (Applied Biosystems) in the $7900 \mathrm{HT}$ Fast Real-Time or the StepOne PCR Systems (Applied Biosystems). Primer sequences are listed below.

Lentiviral vectors for rescue and knockdown. For rescue experiments in Trim $33^{-/-} \mathrm{iM}$, the lentiviral vector was constructed by inserting full-length flag-TRIM33 from the pSG5-flag-TRIM33 (ref. 15) into the pTRIP/DU3-MNDIRES-GFP vector, downstream the MND promoter, using the Gateway technology (Life Technologies). Deletions of the coiled-coil domain (aa 321-457), the ubiquitin ligase activity (aa 141-145) and the SMAD-interaction (aa 555-876) domain were generated by PCR. The pTRIP/ $\triangle \mathrm{U} 3-\mathrm{MND}-\mathrm{GFP}$ was used in control experiments. NIH3T3 PU1 ${ }^{+}$cells were obtained by transducing a lentiviral vector pTRIP/ $\Delta U 3$ MND-PU.1-IRES-GFP. For TRIM33 knocking-down in NIH3T3 or NIH3T3 PU. $1^{+}$, the sequence of the shRNA targeting mouse Trim33 was obtained from Sigma Aldrich ( $5^{\prime}$-CCGGCGGACTTAAATCGGTTGTTAACTCGAGTTAA CAACCGATTTAAGTCCGTTTTTG- $3^{\prime}$ ) and cloned in the pSuper vector (OligoEngine) downstream the $\mathrm{H} 1$ promoter. H1-shRNATRIM33 fragment was then cloned in the pTRIP/ $\Delta \mathrm{U} 3-\mathrm{EF} 1 \alpha-\mathrm{GFP}$, where the GFP coding sequence is under the control of the EF1 $\alpha$ promoter. All the constructs were verified by sequencing. Lentiviral vector production and cells transduction were carried out as described $^{42}$. GFP-positive cells were sorted and used in functional assays.

Luciferase assays. A $391 \mathrm{bp}$ DNA fragment of murine Ifnb1 promoter was obtained by PCR on genomic DNA and cloned into the pGL3 Basic vector (Promega) using KpnI and XhoI sites. A 408 bp DNA fragment spanning the - $15 \mathrm{~kb}$ PU.1/TRIM33-binding site was obtained by PCR of genomic DNA and cloned upstream of the $391 \mathrm{bp}$ DNA fragment of murine Ifnbl promoter into the pGL3 Basic vector. After sequencing of the different constructs, RAW 264.7 cells were transfected using JetPEI (PolyPlus-transfection) with the indicated constructs and the TK-renilla reporter vector (Promega), as an internal control for normalization. Twenty-four hours after transfection, cells were activated with LPS $\left(100 \mathrm{ng} \mathrm{ml}^{-1}\right)$ and luciferase activity was determined using the dual-luciferase reporter assay (Promega). Firefly luciferase activity was normalized with respect to renilla luciferase activity.

Genomic deletions of ICE with the CRISPR/CAS9 system. Two guides RNA (gRNA) were designed to delete a 1,361 bp fragment containing the $-15 \mathrm{~kb}$ region using the CRISPR Design Tool (http://tools.genome-engineering.org), to minimize the number of off-target sites. Sequences for gRNA 5A2 and 3A are $5^{\prime}$-TTC TCGTTCATTGTTAGCGA- $3^{\prime}$ and $5^{\prime}$-CTCTAGTTTAGACGTTTAAC- $3^{\prime}$, respectively. Singles gRNA were then cloned into the LentiCRISPRv2 (Addgene) vector, which contains the human CAS9-coding sequence, using BsmBI cloning sites. Lentivirus were produced and co-transduced into RAW 264.7 and NIH3T3. After puromycin selection ( $3 \mu \mathrm{g} \mathrm{ml}^{-1}$, Sigma Aldrich), cells were clonally isolated by fluorescence-activated cell sorting and individual clones were screened for deletion by PCR using the following primers: F5-1:5'-TGACGACAAATGTGGT

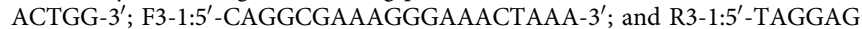
TGGCAGATGGGAAG- $3^{\prime}$

Deletions of 100 or $176 \mathrm{bp}$ were carried out using one gRNA guide $\left(5^{\prime}\right.$-AAA CACGTTAGTTGTCAGAC- $3^{\prime}$ ) flanking the PU.1/TRIM33 site at the ICE and cloned into the LentiCRISPRv2 vector as above. After puromycin selection and clonal isolation, WT and ICE ${ }^{-1-}$ RAW 264.7 cells were identified by PCR using the following primers: F: 5'-AGCAAAGCCGAAGAAGACAC- $3^{\prime}$ and R: $5^{\prime}$-GGA AGAGGACGAGAGAACCA- $3^{\prime}$.

All the clones were verified by sequencing of a $2 \mathrm{~kb}$ genomic region containing the ICE.

Protein extraction and western blot. Total proteins from BMDM or iM were extracted using lysis buffer $(50 \mathrm{mM}$ Tris pH8, $300 \mathrm{mM} \mathrm{NaCl}, 1 \%$ Triton X-100, $0.1 \%$ sodium dodecyl sulfate, 1 mM EDTA, phosphatase inhibitor cocktail-1 and -2 (Sigma Aldrich) and complete protease inhibitor cocktail (Roche)). Proteins were separated on $3-8 \%$ Tris-Acetate gel or $4-12 \%$ Bis Tris gel (Life Technologies), transferred on a nitrocellulose membrane (Amersham) and blotted with the indicated antibodies. Following staining with HRP-coupled secondary antibodies, the proteins were detected with ECL Prime by chemiluminescence (Amersham).

Antibodies. The following antibodies were used in western blot and ChIP: TRIM33 (3E9, Euromedex for WB (1:1,000); A301-059A, Bethyl for ChIP (1:100)) 
c-JUN (sc-1694X, Santa Cruz (1:200)), p65 (sc-372X, Santa Cruz (1:200)), RNA Pol II (sc-899X, Santa Cruz (1:200)), H3K4me3 (07-473, Millipore (1:500)), PU.1 (sc-352X, Santa Cruz (1:10,000 for western blot; 1:500 for ChIP)), p300 (sc-585X, Santa Cruz), AcH3 (06-599, Millipore (1:500)), H3K4me1 (ab-8895, Abcam (1:500)), Flag (M2 monoclonal, Sigma Aldrich (1:1,000)), CBP (A-22, sc-369, Santa Cruz (1:1,000)), $\beta$-actin (A5441, Sigma Aldrich $(1: 10,000)$ ).

Primer sequences. The following primer sequences were used in quantitative RT-PCR, ChIP and 3C-seq: Ifnb1 F: $5^{\prime}$-CTGGCTTCCATCATGAACAA- $3^{\prime}$; Ifnb1 R: $5^{\prime}$-AGAGGGCTGTGGTGGAGAA-3'; Ifna F: 5'-GGATGTGACCTTCCTCAG ACTC-3'; Ifna R: 5'-ACCTTCTCCTGCGGGAATCCAA-3'; Hprt F: 5'-GCTGGT GAAAAGGACCTCT- ${ }^{\prime}$; Hprt R: $5^{\prime}$-CACAGGACTAGAACACCTGC- $3^{\prime}$; Ifnb1 promoter F: $5^{\prime}$-CCACCTGTTGTTCATGATGG-3'; Ifnb1 promoter R: $5^{\prime}$-CATT CTCACTGCAGCCTTTG-3'; Ifnb1 ICE F: 5'-CCAAGGGTTGCGTAATGAAC-3'; Ifnb1 ICE R: 5'-CCCGATCTTCAAATCCAGTC-3'; Ifnb1 + 2,5kb F: 5'-TAGCT TCCATGCCCAGTTTC-3'; Ifnb1 + 2,5 kb R: $5^{\prime}$-CCAAAAGTATGCCCATC ACC- $3^{\prime}$; $\beta$-globin major promoter F: $5^{\prime}$-GAAGCCTGATTCCGTAGAGC-3'; $\beta$-globin major promoter R: $5^{\prime}$-CAACTGATCCTACCTCACCTTATATGC- $3^{\prime}$; ICE EcoRI primer: $5^{\prime}$-AATGATACGGCGACCACCGAACACTCTTTCCCTACA CGACGCTCTTCCGATCT- $3^{\prime}$ followed by a 4 nt tag and $5^{\prime}$-GATGGCACACCTT GAATTC-3'; ICE DpnII primer: $5^{\prime}$-CAAGCAGAAGACGGCATACGAGACGA CAAATGTGGTACTG-3'; Ifnb1 EcoRI primer: $5^{\prime}$-AATGATACGGCGACCACC GAACACTCTTTCCCTACACGACGCTCTTCCGATCT- $3^{\prime}$ followed by a $4 \mathrm{nt}$ tag and 5'-GGGTGTTCTATTTTATCAATAC-3'; Ifnb1 DpnII primer: 5'-CAA GCAGAAGACGGCATACGATTTCTAGAGTTTCCGACTCTG-3'.

\section{References}

1. Trinchieri, G. Type I interferon: friend or foe? J. Exp. Med. 207, 2053-2063 (2010)

2. Stetson, D. B. \& Medzhitov, R. Type I interferons in host defense. Immunity 25, 373-381 (2006).

3. Teijaro, J. R. et al. Persistent LCMV infection is controlled by blockade of type I interferon signaling. Science 340, 207-211 (2013).

4. Wilson, E. B. et al. Blockade of chronic type I interferon signaling to control persistent LCMV infection. Science 340, 202-207 (2013).

5. Ford, E. \& Thanos, D. The transcriptional code of human IFN-beta gene expression. Biochim. Biophys. Acta. 1799, 328-336 (2010).

6. Li, P. et al. IRF8 and IRF3 cooperatively regulate rapid interferon-beta induction in human blood monocytes. Blood 117, 2847-2854 (2011)

7. Kim, H. \& Seed, B. The transcription factor MafB antagonizes antiviral responses by blocking recruitment of coactivators to the transcription factor IRF3. Nat. Immunol. 11, 743-750 (2010).

8. Siednienko, J. et al. Nuclear factor kappaB subunits RelB and cRel negatively regulate Toll-like receptor 3-mediated beta-interferon production via induction of transcriptional repressor protein YY1. J. Biol. Chem. 286, 44750-44763 (2011).

9. Josse, T. et al. Association of the interferon-beta gene with pericentromeric heterochromatin is dynamically regulated during virus infection through a YY1-dependent mechanism. Nucleic. Acids. Res. 40, 4396-4411 (2012)

10. Weill, L., Shestakova, E. \& Bonnefoy, E. Transcription factor YY1 binds to the murine beta interferon promoter and regulates its transcriptional capacity with a dual activator/repressor role. J. Virol. 77, 2903-2914 (2003).

11. Hatakeyama, S. TRIM proteins and cancer. Nat. Rev. Cancer 11, 792-804 (2011)

12. Herquel, B., Ouararhni, K. \& Davidson, I. The TIFlalpha-related TRIM cofactors couple chromatin modifications to transcriptional regulation, signaling and tumor suppression. Transcription 2, 231-236 (2011).

13. Herquel, B. et al. Trim24-repressed VL30 retrotransposons regulate gene expression by producing noncoding RNA. Nat. Struct. Mol. Biol. 20, 339-346 (2013).

14. Xi, Q. et al. A poised chromatin platform for TGF-beta access to master regulators. Cell 147, 1511-1524 (2011).

15. Kusy, S. et al. Adult hematopoiesis is regulated by TIF1gamma, a repressor of TAL1 and PU.1 transcriptional activity. Cell Stem Cell 8, 412-425 (2011).

16. Bai, X. et al. TIF1gamma controls erythroid cell fate by regulating transcription elongation. Cell 142, 133-143 (2010).

17. Ozato, K., Shin, D. M., Chang, T. H. \& Morse, 3rd H. C. TRIM family proteins and their emerging roles in innate immunity. Nat. Rev. Immunol. 8, 849-860 (2008).

18. McNab, F. W., Rajsbaum, R., Stoye, J. P. \& O'Garra, A. Tripartite-motif proteins and innate immune regulation. Curr. Opin. Immunol. 23, 46-56 (2011).

19. Tisserand, J. et al. Tripartite motif 24 (Trim24/Tif1 alpha) tumor suppressor protein is a novel negative regulator of interferon (IFN)/signal transducers and activators of transcription (STAT) signaling pathway acting through retinoic acid receptor alpha (Rar alpha) inhibition. J. Biol. Chem. 286, 33369-33379 (2011)
20. Weng, L. et al. The E3 ubiquitin ligase tripartite motif 33 is essential for cytosolic RNA-induced NLRP3 inflammasome activation. J. Immunol. 193, 3676-3682 (2014).

21. Squadrito, M. L. et al. Endogenous RNAs modulate microRNA sorting to exosomes and transfer to acceptor cells. Cell Rep. 8, 1432-1446 (2014).

22. Banerjee, A. R., Kim, Y. J. \& Kim, T. H. A novel virus-inducible enhancer of the interferon-beta gene with tightly linked promoter and enhancer activities. Nucleic Acids Res. 42, 12537-12554 (2014).

23. Uhlenhaut, N. H. et al. Insights into negative regulation by the glucocorticoid receptor from genome-wide profiling of inflammatory cistromes. Mol. Cell 49, 158-171 (2013).

24. Lara-Astiaso, D. et al. Immunogenetics. Chromatin state dynamics during blood formation. Science 345, 943-949 (2014).

25. Bowers, E. M. et al. Virtual ligand screening of the p300/CBP histone acetyltransferase: identification of a selective small molecule inhibitor. Chem. Biol. 17, 471-482 (2010)

26. Ng, C. T. et al. Blockade of interferon Beta, but not interferon alpha, signaling controls persistent viral infection. Cell Host Microbe 17, 653-661 (2015).

27. Hogner, K. et al. Macrophage-expressed IFN-beta contributes to apoptotic alveolar epithelial cell injury in severe influenza virus pneumonia. PLoS Pathog. 9, e1003188 (2013)

28. Ghavi-Helm, Y. et al. Enhancer loops appear stable during development and are associated with paused polymerase. Nature 512, 96-100 (2014).

29. Jin, F. et al. A high-resolution map of the three-dimensional chromatin interactome in human cells. Nature 503, 290-294 (2013).

30. Pattanayak, V., Guilinger, J. P. \& Liu, D. R. Determining the specificities of TALENs, Cas9, and other genome-editing enzymes. Methods Enzymol. 546, 47-78 (2014).

31. Wang, X. et al. Unbiased detection of off-target cleavage by CRISPR-Cas9 and TALENs using integrase-defective lentiviral vectors. Nat. Biotechnol. 33, 175-178 (2015).

32. Kim, S., Kim, D., Cho, S. W., Kim, J. \& Kim, J. S. Highly efficient RNA-guided genome editing in human cells via delivery of purified Cas9 ribonucleoproteins. Genome Res. 24, 1012-1019 (2014)

33. Mancino, A. et al. A dual cis-regulatory code links IRF8 to constitutive and inducible gene expression in macrophages. Genes Dev. 29, 394-408 (2015).

34. Rajsbaum, R. et al. Unanchored K48-linked polyubiquitin synthesized by the E3-ubiquitin ligase TRIM6 stimulates the interferon-IKKepsilon kinasemediated antiviral response. Immunity 40, 880-895 (2014).

35. Inn, K. S. et al. Linear ubiquitin assembly complex negatively regulates RIG-Iand TRIM25-mediated type I interferon induction. Mol. Cell 41, 354-365 (2011)

36. Higgs, R. et al. The E3 ubiquitin ligase Ro52 negatively regulates IFN-beta production post-pathogen recognition by polyubiquitin-mediated degradation of IRF3. J. Immunol. 181, 1780-1786 (2008).

37. Tsuchida, T. et al. The ubiquitin ligase TRIM56 regulates innate immune responses to intracellular double-stranded DNA. Immunity 33, 765-776 (2010).

38. Merika, M., Williams, A. J., Chen, G., Collins, T. \& Thanos, D. Recruitment of $\mathrm{CBP} / \mathrm{p} 300$ by the IFN beta enhanceosome is required for synergistic activation of transcription. Mol. Cell 1, 277-287 (1998).

39. Yamamoto, H., Kihara-Negishi, F., Yamada, T., Hashimoto, Y. \& Oikawa, T. Physical and functional interactions between the transcription factor PU.1 and the coactivator CBP. Oncogene 18, 1495-1501 (1999).

40. Bai, Y., Srinivasan, L., Perkins, L. \& Atchison, M. L. Protein acetylation regulates both PU.1 transactivation and Ig kappa 3' enhancer activity. J. Immunol. 175, 5160-5169 (2005).

41. Stadhouders, R. et al. Multiplexed chromosome conformation capture sequencing for rapid genome-scale high-resolution detection of long-range chromatin interactions. Nat. Protoc. 8, 509-524 (2013).

42. Kusy, S. et al. NKX3.1 is a direct TAL1 target gene that mediates proliferation of TAL1-expressing human T cell acute lymphoblastic leukemia. J. Exp. Med. 207, 2141-2156 (2010)

43. Heinz, S. et al. Simple combinations of lineage-determining transcription factors prime cis-regulatory elements required for macrophage and B cell identities. Mol. Cell 38, 576-589 (2010).

\section{Acknowledgements}

We are grateful to the staff of the iRCM animal facility for excellent support during the mouse studies and to N. Dechamps and J. Baijer of the iRCM cytometry platform for excellent support during FACS experiments. F.F. and A.P. are supported by fellowships from Dim Stem Pole and La Ligue contre le Cancer. This project was supported by grants to P.-H.R. from La Ligue contre le Cancer (équipe labelisée), ANR (ANR-12-BSV1-0001-02), INCA (PL BIO 013031), Inserm, CEA/DSV, Paris-Diderot and Paris-Sud.

\section{Author contributions}

P.-H.R., F.F., I.D. and G.R. designed the project, analysed data and wrote the manuscript. A.P., V.P., A.-S.G., F.F., G.R., M.D. and D.L. organized, performed and analysed most experiments and wrote the manuscript. E.S., A.v.d.H. and P.K. assisted with experimental 
design and data analysis for 3C-Seq and wrote the manuscript. M.L.S. and M.D.P. assisted with experimental design for iM production and wrote the manuscript. All authors approved the final version of the manuscript.

\section{Additional information}

Accession codes: High throughput sequencing data have been deposited in the Gene Expression Omnibus (GEO) database (www.ncbi.nlm.nih.gov/geo), under accession codes GSE73322 (3c-seq) and GSE43654 (ChIP-seq).

Supplementary Information accompanies this paper at http://www.nature.com/ naturecommunications

Competing financial interests: The authors declare no competing financial interests.
Reprints and permission information is available online at http://npg.nature.com/ reprintsandpermissions/

How to cite this article: Ferri, F. et al. TRIM33 switches off Ifnbl gene transcription during the late phase of macrophage activation. Nat. Commun. 6:8900 doi: 10.1038/ncomms9900 (2015).

(c) (i) This work is licensed under a Creative Commons Attribution 4.0 International License. The images or other third party material in this article are included in the article's Creative Commons license, unless indicated otherwise in the credit line; if the material is not included under the Creative Commons license, users will need to obtain permission from the license holder to reproduce the material. To view a copy of this license, visit http://creativecommons.org/licenses/by/4.0/ 